\title{
Numerical Solution of the Full Potential Equation Using a Chimera Grid Approach
}

\section{Terry L. Holst}

July 1995

(NASA-TM-110360) NUHERICAL SOLUTION OF THE FULL POTENTIAL EQUATION USING A CHIMERA GRID APPROACH (NASA. Ames Research Center) $33 \mathrm{p}$
N95-32189

Unclas
National Aeronautics and Space Administration 

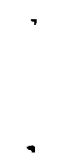

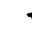




\section{Numerical Solution of the Full Potential Equation Using a Chimera Grid Approach}

Terry L. Holst, Ames Research Center, Moffett Field, California

July 1995

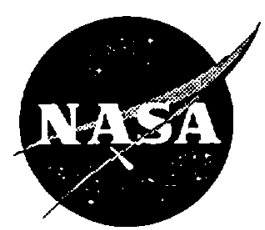

National Aeronautics and Space Administration 
. 


\title{
NUMERICAL SOLUTION OF THE FULL POTENTIAL EQUATION USING A CHIMERA GRID APPROACH
}

\author{
Terry L. Holst \\ Ames Research Center
}

\section{SUMMARY}

A numerical scheme utilizing a chimera zonal grid approach for solving the full potential equation in two spatial dimensions is described. Within each grid zone a fully-implicit approximate factorization scheme is used to advance the solution one iteration. This is followed by the explicit advance of all common zonal grid boundaries using a bi-linear interpolation of the velocity potential. The presentation is highlighted with numerical results simulating the flow about a two-dimensional, nonlifting circular cylinder. For this problem, the flow domain is divided into two parts; an inner portion covered by a polar grid and an outer portion covered by a Cartesian grid. Both incompressible and compressible (transonic) flow solutions are included. Comparisons made with an analytic solution as well as single grid results indicate that the chimera zonal grid approach is a viable technique for solving the full potential equation.

\section{INTRODUCTION}

Numerous computational fluid dynamics (CFD) simulations have been performed for the flow about complex aerodynamic shapes including reasonably complete aircraft in the past several years. Many numerical schemes have been used for these simulations including formulations based on the full potential, Euler and Navier-Stokes equations utilizing a variety of zonal and unstructured grid approaches. Many of these simulation capabilities have found their way into the aerospace vehicle design environment where they are typically used to complement wind tunnel testing associated with aerodynamic design. Despite the remarkable growth of CFD simulations in the aerospace vehicle design environment, there are three major areas that need significant research attention before the ultimate potential of CFD simulations can be realized. These three areas include turbulence and transition modeling, problem setup automation, and job execution efficiency. $\S$ The key requirement in the turbulence and transition area is development of turbulence and transition models that have increased accuracy and reliability with an increased breadth of application. These new models must provide accurate prediction of a flow's viscous effects (e.g., drag) across a range of conditions including wind-tunnel-to-flight Reynolds number variations. This complex research area is focused on all flow regimes, but is especially difficult for separated or "edge of the envelope" flows. The area of turbulence and transition is an extremely important area of research in fluid dynamics, but is beyond the scope of the present study and will not be discussed further.

The second area requiring a significant amount of attention is associated with problem setup automation, i.e., reducing the time it takes to input and massage the geometry definition so as to make it compatible with the CFD solver being used. This time is typically characterized by many labor hours in front of a workstation with little automation (at least for complex full aircraft geometries). Increased automation in this area could reduce problem setup time by an order of magnitude or more. In addition, automation of geometry definition and modification would significantly enhance abilities associated with aerodynamic numerical optimization.

The third and final area of required attention in CFD simulations is job execution efficiency. Reducing time in this area is important to reduce computer costs and to produce results with less wall clock time,

$\S$ A fourth major area requiring additional research attention is the integration of multidiscipline simulation capabilities including such disciplines as aerodynamics, structural dynamics, controls, propulsion, design optimization, etc. However, this topic will not be discussed further in this report. 
especially when many results are required for parametric analysis. Typical three-dimensional simulations involving numerical solution of the steady Navier-Stokes equations require from several hours to several tens of hours of execution time per case on the fastest super computers, e.g., the Cray C-90. Unsteady simulations are even more expensive by an order of magnitude or more. Thus, parametric analysis with such an approach is extremely expensive in both wall clock time and computer costs and is not even feasible for many of the complex geometry applications that exist in the aerospace vehicle design environment. Improvement in this area can be-accomplished in several ways including faster more cost-effective computer hardware; faster algorithms; more efficient programming, i.e., more efficient vectorization or parallelization; a more judicious choice of qoverning equation formulation, i.e., utilization of the more complete Navier-Stokes formulation only when required and more approximate formulations when appropriate; or a combination of all of the above. Efficiency issues are even more important now with the transition of the aerospace industry from mainframe computers to generally more numerous but slower workstation-class computing hardware.

It is primarily in the last category that the present research activity will have its impact. The present long term objective is to develop a chimera-based full potential flow solver which will be compatible with the well-established OVERFLOW Euler/Navier-Stokes flow solver developed at NASA Ames. 1 Thus, the user will have an option of which flow solver to use in the chimera-based zonal grid approach: full potential, Euler or Navier-Stokes. Of course, the full potential option will not be applicable for all applications, but for those that are applicable, the execution time should be up to two orders of magnitude less than for the Navier-Stokes formulation. Indeed, a chimera-based full potential solver should have modest execution times on even moderate-speed workstations. In a parametric study the bulk of the required computations could utilize the full potential approach and then a few selected conditions could be "checked" with a more complete, and thus more accurate, Euler or Navier-Stokes simulation. Such an approach would be extremely cost effective especially considering that all of these approaches would utilize the same problem setup and post processing software and to a large extent the same grid generation software. Applications of this new approach are quite numerous and include providing a fast mechanism for assessing wind tunnel wall and support interference effects associated with wind tunnel testing. This could be directly utilized to support the IofNEWT (Integration of Numerical Experimental Wind Tunnel) Project being implemented at NASA Ames to integrate CFD into wind tunnel testing or it could be used directly in the industrial preliminary design environment.

A more detailed description of the overset or chimera zonal grid approach including a brief review of past work is discussed next. In the chimera zonal grid approach a separate boundary conforming grid is generated about each major feature of a geometry. For example, for a transport aircraft consisting of a wing/body/pylon/nacelle a total of five grid zones might be required, one for the near field surrounding each of the major geometric features (wing, body, pylon, and nacelle) and a fifth background grid to "connect" the near field grid zones to freestream. Each of the component grids is generated without regard to any of the other component grids. Each boundary grid point receives its boundary condition information from either freestream, flow tangency (assuming the solver is inviscid), symmetry, or from another component grid using general interpolation. Some of the grid cells generated for one component of the geometry may have grid points that lie inside other components of the geometry. Computations at these points are handled by an "IBLANK array multiplier" which has a value associated with each grid point. IBLANK is equal to one for points in valid flow regions and to zero for points in invalid or "blanked out" flow regions. Computations proceed in an identical fashion at all grid points, which permits efficient code vectorization, but because of the IBLANK array multiplication, only grid points in valid regions of flow get updated as the iteration proceeds.

Early work in the development of the multiple-zone overset or chimera approach can be found in Refs. 2-5. In these references the basic concepts of the approach were developed with applications consisting of relatively simple two and three-dimensional simulations. The approach was further developed and applied to a large variety of complex geometry applications, which (of course) is the real purpose of the methodology. A few examples of chimera applications are given in Refs. 6-8 for Space Shuttle ascent-mode simulations, Refs. $9-13$ for F-18 aircraft simulations at large angles of attack with and without propulsion effects, Ref. 14 for a simulation of the flow about the AV-8B Harrier in near hover conditions above a ground plane, and Rets. 15-23 for a variety of applications involving 
unsteady flows, most containing one body moving relative to another. Indeed, the chimera approach has been used to provide valuable aerodynamic data for many important applications.

In all the above examples, the Euler or Navier-Stokes governing equations were used. Very few examples of a chimera approach exist in the literature for solving the full potential equation. One early example is Atta and Vadyak ${ }^{24}$ where a chimera-like approach was used to solve an inviscid flow about a wing-nacelle configuration. In addition, there is not much information on error analysis associated with the chimera approach. The questions of how much error does the interpolation process produce and what is the effect of this error on various aspects of the solution away from the interface boundary, have not been generally addressed. One notable exception to this is the work of Meakin ${ }^{25}$ where a chimera scheme error analysis was performed for a number of steady and unsteady transonic airfoil cases. Results from this study demonstrated the viability of the chimera approach for the Euler formulation being used. The purpose of the present study is to investigate the above two questions in the context of a chimera full potential solver. The presentation begins with a discussion of the governing equation formulation and the numerical scheme used. Next, computational results for the flow about a two-dimensional circular cylinder are presented for both incompressible and compressible cases. Comparison with single grid results and analytic solutions (incompressible cases only) are made to establish levels of accuracy for the scheme. Finally, the presentation ends with concluding remarks.

\section{GOVERNING EQUATION FORMULATION}

The steady, two-dimensional, full potential equation written in strong conservation-law form is given by

$$
\begin{gathered}
\left(\rho \phi_{x}\right)_{x}+\left(\rho \phi_{y}\right)_{y}=0 \\
\rho=\left[1-\frac{\gamma-1}{\gamma+1}\left(\phi_{x}^{2}+\phi_{y}^{2}\right)\right]^{\frac{1}{\gamma-1}}
\end{gathered}
$$

where $\rho$ is the fluid density, $x$ and $y$ are Cartesian coordinates, $\gamma$ is the ratio of specific heats, and $\phi$ is the full or exact velocity potential defined by

$$
\nabla \phi=\vec{q}
$$

where $\vec{q}$ is the local velocity vector. The mere existence of the velocity potential implies that the curl of the velocity vector must vanish, i.e.

$$
\nabla \times \vec{q}=0
$$

Thus, flows governed by the full potential equation must be irrotational. In addition, derivation of the density relation [Eq. (1b) above] requires the assumption of isentropic flow. In Eqs. (1) the density $(\rho)$ and velocity components $\left(\phi_{x}\right.$ and $\phi_{y}$ ) are nondimensionalized by the stagnation density $\left(\rho_{s}\right)$ and the critical speed of sound (a.), respectively. Additional relations valid for these flow assumptions and this nondimensionalization include

Isentropic equation of state:

$$
\frac{p}{\rho^{\gamma}}=\frac{\gamma+1}{2 \gamma}
$$


Bernoulli's equation:

Speed of sound definition:

$$
\frac{q^{2}}{2}+\frac{a^{2}}{\gamma-1}=\frac{1}{2} \frac{\gamma+1}{\gamma-1}
$$

$$
a^{2}=\gamma \frac{p}{\rho}
$$

where $p$ is the fluid pressure, $a$ is the local speed of sound, and $q$ is the magnitude of the local fluid velocity.

To complete the above system boundary conditions are required at freestream and the geometry surface. The freestream boundary condition, simply stated, is given by

$$
x^{2}+y^{2} \rightarrow \infty, \quad \phi \rightarrow \phi_{\infty}
$$

where $\phi_{\infty}$ is the freestream distribution of the velocity potential, usually uniform flow. The surface boundary condition is that of flow tangency given by

$$
\vec{q} \cdot \vec{n}=0
$$

where $\vec{n}$ is a unit vector normal to the geometry of interest.

Equation (1) expresses mass conservation for flows that are isentropic and irrotational. Despite these limiting assumptions, the full potential formulation can be used in a shock-capturing context providing the shock waves are weak. The corresponding shock-jump conditions are valid approximations to the Rankine-Hugoniot shock jump conditions (derived from the Euler equations) for many applications. The key parameter in this situation is the normal component of the Mach number just upstream of the shock wave in question, which must remain below about 1.3 for the full potential formulation to be a reasonable approximation to the Euler equations. This is well within the scope of many transonic flow applications and includes the cruise conditions for most transonic transport aircraft. More discussion on this point including a comparison of the Euler and isentropic full potential shock polars is presented in Steger and Baldwin. 26

Equation (1) is transformed from the physical domain (Cartesian coordinates) into the computational domain using a general, independent-variable transformation. This general transformation, indicated by

$$
\begin{aligned}
& \xi=\xi(x, y) \\
& \eta=\eta(x, y)
\end{aligned}
$$

maintains the strong conservation-law form of Eqs. (1). The final transformed version of the full potential equation is given by

$$
\begin{gathered}
\left(\frac{\rho U}{J}\right)_{\xi}+\left(\frac{\rho V}{J}\right)_{\eta}=0 \\
\rho=\left[1-\frac{\gamma-1}{\gamma+1}\left(U_{\phi_{\xi}}+V \phi_{\eta}\right)\right]^{\frac{1}{\gamma-1}}
\end{gathered}
$$


where

$$
\begin{aligned}
& U=A_{1} \phi_{\xi}+A_{2} \phi_{\eta} \\
& V=A_{2} \phi_{\xi}+A_{3} \phi_{\eta} \\
& A_{1}=\nabla \xi \bullet \nabla \xi=\xi_{x}^{2}+\xi_{y}^{2} \\
& A_{2}=\nabla \xi \bullet \nabla \eta=\xi_{x} \eta_{x}+\xi_{y} \eta_{y} \\
& A_{3}=\nabla \eta \bullet \nabla \eta=\eta_{x}^{2}+\eta_{y}^{2} \\
& J=\xi_{x} \eta_{y}-\xi_{y} \eta_{x}=\left(x_{\xi} y_{\eta}-x_{\eta} y_{\xi}\right)^{-1}
\end{aligned}
$$

In Eqs. (4), $U$ and $V$ are the contravariant velocity components along the $\xi$ and $\eta$ coordinate directions, respectively; $A_{1}, A_{2}$ and $A_{3}$ are metric quantities; and $J$ is the determinant of the transformation Jacobian. The above full potential governing equation formulation can be used for general geometries in which the aerodynamic surface of interest is mapped to a constant coordinate line in the computational domain. This makes the flow-tangency boundary condition easy and accurate to implement. In transformed coordinates the flow-tangency boundary condition becomes

$$
\begin{aligned}
& \vec{q} \cdot \nabla \eta=0 \\
& \vec{q} \cdot \nabla \eta=\left(\phi_{x} \vec{i}+\phi_{y} \vec{j}\right) \cdot\left(\eta_{x} \vec{i}+\eta_{y} \vec{j}\right)=V \\
& V=0
\end{aligned}
$$

More simply stated, the contravariant velocity component in the $\eta$-direction must vanish at the surface where flow tangency is required. In the above case, it is assumed that an $\eta=$ constant coordinate line has been mapped to the aerodynamic surface of interest.

\section{NUMERICAL APPROACH}

\section{Spatial differencing scheme}

A second-order-accurate spatial differencing approximation to the full potential equation written in general $\xi-\eta$ coordinates is given by

$$
\stackrel{\leftarrow}{\delta}_{\xi}\left(\frac{\rho U}{J}\right)_{i+1 / 2, j}+\overleftarrow{\delta}_{\eta}\left(\frac{\rho V}{J}\right)_{i, j+1 / 2}=0
$$

where

$$
\begin{aligned}
U_{i+1 / 2, j}= & A_{1_{i+v 2, j}}\left(\phi_{i+1, j}-\phi_{i, j}\right) \\
& +\frac{1}{4} A_{2_{i+12, j}}\left(\phi_{i+1, j+1}-\phi_{i+1, j-1}+\phi_{i, j+1}-\phi_{i, j-1}\right) \\
V_{i, j+1 / 2}= & \frac{1}{4} A_{2_{i, j+1 / 2}}\left(\phi_{i+1, j+1}-\phi_{i-1, j+1}+\phi_{i+1, j}-\phi_{i-1, j}\right) \\
& +A_{3_{i, j+12}}\left(\phi_{i, j+1}-\phi_{i, j-1}\right)
\end{aligned}
$$


The operators

$$
\overleftarrow{\delta}_{\xi} \text { and } \stackrel{\leftarrow}{\delta}_{\eta}
$$

are backward difference operators in the $\xi$ - and $\eta$-coordinate directions, respectively, and are defined by

$$
\begin{aligned}
& \stackrel{\leftarrow}{\delta}_{\xi}(-)_{i, j}=\left({ }_{-}\right)_{i, j}-\left({ }_{-}\right)_{i-1, j} \\
& \stackrel{\leftarrow}{\delta}_{\eta}(-)_{i, j}=(-)_{i, j}-(-)_{i, j-1}
\end{aligned}
$$

The $i$ and $j$ subscripts used in the Eqs. (5)-(7) are used to denote position in the finite-difference grid such that $\xi=i \Delta \xi$ and $\eta=i \Delta \eta$. For convenience, the $\Delta \xi$ and $\Delta \eta$ values are taken to be unity.

Values of $\rho, A_{1}, A_{2}, A_{3}$, and $J$ are computed using the freestream preserving spatial differencing scheme described by Flores et al. ${ }^{27}$ and Thomas and Holst. ${ }^{28}$ In particular, the density is computed at half points in the $\xi$-direction, i.e., at $i+1 / 2, j$, using Eq. (3b). All derivatives appearing in this formula, both for the velocity potential and the mapping metrics, are evaluated using

$$
\begin{aligned}
& \left.r_{\xi}\right|_{i+1 / 2, j} \cong(-)_{i+1, j}-(-)_{i, j} \\
& \left.\left.r_{\eta}\right|_{j+1 / 2, j} \cong \frac{1}{4}\left[(-)_{i, j+1}-(-)_{i, j-1}+\left({ }_{-}\right)_{i+1, j+1}-\left({ }_{-}\right)_{i+1, j-1}\right)\right]
\end{aligned}
$$

where $r$ is equal to $\phi, x$, or $y$, as appropriate. Of course, for the incompressible computations presented herein, the density can simply be replaced by a constant.

At supersonic points the centrally-differenced, second-order-accurate spatial discretization scheme just presented must be upwind biased to maintain stable operation. This is accomplished by replacing the density in the $\xi$-flux computation with an upwind biased value of the density. Thus, the new spatial differencing scheme is given by

$$
\overleftarrow{\delta}_{\xi}\left(\frac{\tilde{\rho} U}{J}\right)_{i+1 / 2, j}+\overleftarrow{\delta}_{\eta}\left(\frac{\rho V}{J}\right)_{i, j+1 / 2}=0
$$

where the density coefficient $\tilde{\rho}_{i+1 / 2, j}$ is defined by

$$
\tilde{\rho}_{i+1 / 2, j}=\rho_{i+1 / 2, j}+v_{i+1 / 2, j}\left(\rho_{i-1 / 2, j}-\rho_{i+1 / 2, j}\right)
$$

and

$$
v_{i+1 / 2, j}=\left\{\begin{array}{ccc}
2.46625\left(2 \rho^{*}-\rho_{i+1 / 2, j}-\rho_{i-1 / 2, j}\right) C & \text { if } & \rho_{i, j} \leq \rho^{*} \\
0 & \text { if } & \rho_{i, j}>\rho^{*}
\end{array}\right.
$$

The quantity $\rho^{*}$ is a constant equal to the sonic value of the density, which for $\gamma=1.4$, is $0.6339382 \ldots$. The quantity $C$ is a user specified coefficient usually set near a value of one, and $v_{i+1 / 2, j}$ is a switching parameter that controls the amount of upwinding that exists in the numerical 
scheme. Equation (10) is designed such that the value of $v_{i+1 / 2, j}$ will be zero at all subsonic grid points, i.e., a second-order-accurate, central-differenced scheme is retained for subsonic regions of the flow domain, and larger than zero at all supersonic grid points, i.e., a first-order-accurate upwind scheme is utilized for supersonic regions of flow. The supersonic branch of Eq. (10) approximates $\left(M_{i, j}^{2}-1\right) C$, and thus, the amount of upwinding increases dramatically as the extent of supersonic flow increases. To keep the value of $\bar{\rho}_{i+1 / 2, j}$ bounded by $\rho_{i+1 / 2, j}$ and $\rho_{i-1 / 2, j}$, the value of $v_{i+1 / 2, j}$ is constrained to be less than or equal to one.

The supersonic spatial differencing scheme represented by Eqs. (8)-(10) is not valid for arbitrary orientations of a curvilinear coordinate system. This is because upwinding of the density is utilized along only the $\xi$-coordinate direction. Nevertheless, the present scheme is valid for supersonic flow for which the local flow direction is approximately aligned with the positive $\xi$-coordinate direction. Thus, the present scheme is suitable for the purpose at hand, namely, evaluation of a chimera-based zonal grid approach for solving the full potential equation. $\S$

\section{Boundary conditions}

Flow tangency and symmetry plane boundary conditions (as described above) require that the velocity component normal to the applicable boundary must vanish. For a general nonorthogonal mapping such as that described by Eq. (2), the general condition for flow tangency requires the $\eta$ contravariant velocity component at $\eta=\eta_{\min }$ (for example) be zero (i.e., $V_{\eta=\eta_{\min }}=0$ ). This is implemented in the present study using a mass-flux reflection condition given by

$$
\left(\frac{\rho V}{J}\right)_{i, 1 / 2}=-\left(\frac{\rho V}{J}\right)_{i, 3 / 2}
$$

where $j=1$ corresponds to the tangency or symmetry plane surface (i.e., $\eta=\eta_{\min }$ ). In other expressions where $\phi_{\eta}$ is required at $j=1$ [e.g., in Eq. (6) or in the density computation at $j=1$ ], the $V_{\eta=\eta_{\min }}=0$ boundary condition is used again to obtain

$$
\left.\phi_{\eta}\right|_{\eta=\eta_{\min }}=-\frac{A_{2}}{A_{3}} \phi_{\xi}
$$

Thus, a value of $\phi_{\eta}$ at the boundary condition surface can be obtained without using one-sided differences on the velocity potential.

The problem being solved in the present study is that of uniform flow over a circular cylinder. Several different freestream Mach numbers are investigated ranging from incompressible $\left(M_{\infty}=0\right)$ to compressible freestream Mach numbers that yield shocked transonic flow fields. Outer computational domain boundary conditions are of two types, one implemented for incompressible cases and the other for compressible cases. For incompressible cases, the exact solution given by

$$
\phi(x, y)=q_{\infty}\left(1+\frac{R^{2}}{x^{2}+y^{2}}\right) x
$$

is applied at the outer boundary. In Eq. (13) $R$ is the cylinder radius and $q_{\infty}$ is the magnitude of the freestream flow, which in this case is aligned with the $x$ axis. For incompressible cases, because of

$\S$ Generalization of the present scheme for arbitrary orientations of the curvilinear coordinate system is easy to accomplish. See refs. $29-30$ for examples. 
the exact outer boundary condition, location of the outer boundary is not important, and thus, is placed only about four diameters away from the circular cylinder.

For the compressible computations a standard freestream outer boundary condition is used which is given by

$$
\phi(x, y)=q_{\infty} x
$$

For the compressible cases the outer boundary placement is much more important as the freestream boundary condition given by Eq. (14) is technically only valid at infinity. For these computations an outer boundary location near 18 diameters is used.

\section{Iteration scheme}

The iteration scheme utilized in the present study is a modified version of the AF2 iteration scheme that is described in Holst. ${ }^{29}$ Implementation of the scheme is achieved by writing it in a two-step form given by

Step 1:

$$
\left(\alpha-1 B_{i, j} \vec{\delta}_{\xi} R_{i}\right) f_{i, j}^{n}=1 B_{i, j} \alpha \omega L \phi_{i, j}^{n}
$$

Step 2:

$$
\left(\alpha \overleftarrow{\delta}_{\xi}^{*}-\mid B_{i, j} \overleftarrow{\delta}_{\eta} s_{j} \vec{\delta}_{\eta}\right) C_{i, j}^{n}=f_{i, j}^{n}
$$

where the $n$ superscript is an iteration index, $\omega$ is a relaxation parameter (set equal to 1.75 for all cases), $L \phi_{i, j}^{n}$ is the $n$th iteration residual defined by Eq. (8), $f_{i, j}^{n}$ is an intermediate result array stored at each grid point in the finite-difference mesh, and $R_{i}$ and $S_{j}$ are coefficients defined by

$$
R_{i}=\left(\frac{\tilde{\rho} A_{1}}{J}\right)_{i-1 / 2, j}^{n}, \quad S_{j}=\left(\frac{\rho A_{3}}{J}\right)_{i, j+1 / 2}^{n}
$$

In step 1, the $f$ array is obtained by solving a simple bidiagonal matrix equation for each $\eta=$ constant line. The correction array $\left(C_{i, j}^{n}=\phi_{i, j}^{n+1}-\phi_{i, j}^{n}\right)$ is then obtained in the second step from the $f$ array by solving a tridiagonal matrix equation for each $\xi=$ constant line. Note that this version of the AF2 scheme has the $\xi$-direction difference split between the two steps. The Ref. 29 AF2 scheme had the $\eta$-direction difference split between the two steps and represents one of the modifications in the present scheme. The $\xi$-direction splitting generates a $\phi_{\xi t}$-like term, which is useful to the iteration scheme as time-like dissipation primarily in supersonic regions of flow. [The iterative process can be considered as an iteration in pseudotime. Thus, the time-like derivative is introduced by $\left({ }_{-}\right)^{n+1}-\left({ }_{-}\right)^{n} \approx\left({ }_{-}\right)_{t}$.] The $\xi$-direction splitting also places a sweep direction restriction on both steps, namely, in the negative $\xi$-direction (upstream) for the first step and in the positive $\xi$-direction (downstream) for the second step. No sweep direction limitations are placed on either of the two steps due to flow direction.

In Eqs. (15) the quantity $1 B_{i, j}$ is the traditional "IBLANK" integer array used with chimera zonal grid schemes. This quantity is equal to one for all grid points in a particular grid zone that are "activated" or located inside the flow domain. Inactive grid points, e.g., grid points that lie inside a portion of the geometry and therefore outside of the flow domain, are assigned an $I B_{i, j}$ value of zero. The computational algorithm is implemented at all points, but only the $I B=1$ points produce a meaningful 
solution. The influence of all "blanked out" points, i.e., points with $I B=0$, is removed by the iblank array. In Eq. (15b) the backward $\xi$-difference has a special definition for the present chimera full potential scheme which is given by

$$
\overleftarrow{\delta_{\xi}^{*}}()_{i, j}=\left({ }_{-}\right)_{i, j}-1 B_{i, j}\left({ }_{-}\right)_{i-1, j}
$$

This backward difference reduces to the standard definition given by Eq. (7) for all activated grid points that lie inside the flow domain. For grid points that lie outside the flow domain the AF2 scheme given above simply reduces to $C_{i, j}^{n}=0$.

The quantity $\alpha$ introduced in Eqs. (15) is an acceleration parameter and can be considered as $\Delta t^{-1}$. This direct analogy to time provides one strategy for obtaining fast convergence, namely, advance time as fast as possible with large time steps, i.e., small values of $\alpha$. As pointed out in Ballhaus et al., ${ }^{31}$ this is effective for attacking the low-frequency errors but not the high frequency errors. The best overall approach is to use an $\alpha$ sequence containing several values of $\alpha$. The small values are particularly effective for reducing the low-frequency error, and the large values are particularly effective for reducing the high-frequency error. A suitable $\alpha$ sequence used in Refs. 27-31 is given by

$$
\alpha_{k}=\alpha_{H}\left(\frac{\alpha_{L}}{\alpha_{H}}\right)^{\frac{k-1}{M-1}}, \quad k=1,2, \cdots, M
$$

where $M$ is the number of elements in the sequence (a value of eight was used for all cases in the present study), $\alpha_{H}$ is the high-frequency error limit for the sequence (largest value of $\alpha$ ), and $\alpha_{L}$ is the low-frequency error limit (smallest value of $\alpha$ and most sensitive parameter affecting iteration stability and convergence). For the present chimera approach it was found that improved overall convergence would be obtained if each value in the $\alpha$ sequence from the (usually) coarser outer grid was multiplied by a constant just below one (a typical value used in the present study was 0.9 ). This had the effect of increasing the time step for the outer grid relative to the inner finer grid and probably was successful because the high-frequency error content in the outer grid was much smaller relative to the inner finer grid. Typical values in the present study for $\alpha_{L}$ and $\alpha_{H}$ were 0.3 and 6.0, respectively. These values produced stable results for most of the cases presented, but should not be considered to be optimal.

In the present implementation of the AF2 iteration scheme special implicit boundary conditions are required for each sweep, i.e., for each set of matrix inversions. For the first sweep at $i=i_{\max }$ a special boundary condition is required on $f$, which is supplied by a zero slope condition given by

$$
\left.f_{\xi}\right|_{i_{\max }, j} ^{n} \approx f_{i_{\max }+h j}^{n}-f_{i_{\max }-1, j}^{n}=0
$$

At the $j=j_{\max }$ boundary, sweep 2 requires an implicit boundary condition on the correction, which is simply satisfied by setting the correction to zero at this location. At the $j=j_{\min }$ boundary, a similar implicit boundary condition is required on the correction and is supplied by a zero slope condition at this location. These implicit boundary conditions do not affect the final solution's accuracy, but do have a dramatic affect on iteration stability and convergence. For example, the zero slope boundary condition on $f$ creates a stability limitation on $\alpha$ in the present application which can slow convergence dramatically (see South et al. ${ }^{32}$ for more information on this point). This implicitboundary-condition-induced stability limitation can be greatly ameliorated by locally limiting the value of $\alpha$ in the vicinity of the $i=i_{\max }$ boundary without harming overall solution convergence, even for the chimera zonal grid cases. 
Chimera boundary conditions at gird-to-grid zonal interfaces are implemented using bilinear interpolation on the velocity potential. This is the same type of iterpolative interface employed in typical Euler or Navier-Stokes formulations utilizing the chimera approach. That is, both approaches utilize bilinear interpolation (trilinear in three spatial dimensions) on the scheme's dependent variables. For the present full potential formulation the velocity potential is used, and typically for the Euler/Navier-Stokes approach the standard conserved variables in the $Q$ vector are used. Since the velocity potential is a very well behaved function, e.g., it is continuous across shock waves (whereas the Euler/Navier-Stokes $Q$-vector variables generally are not), interpolation of this function is also well behaved. However, most important quantities in fluid dynamics and aerodynamics, e.g., density, pressure, velocity, are only obtained from the velocity potential by taking derivatives. Since numerical differentiation is an error aggravating process, it is expected that some error amplification may exist along interface boundaries for variables such as pressure and velocity.

A schematic of the overset or chimera interpolation procedure is shown in Fig. 1 where fringe points (grid points needing interpolated information) and donor cells (grid cells supplying interpolated information) are both displayed. In constructing the difference scheme for the full potential equation care must be taken to avoid computational stencils that overlap from the interior grid points past the interpolated fringe points and into a region of the grid that is "blanked out." One example of where this occurs in the present scheme is in the calculation of density in the $\eta$-direction flux, namely $\rho_{i, j+1 / 2}$. Normally this value of density is computed from the density array (with values stored at $i+1 / 2, j$ ) using a standard average given by

$$
\rho_{i, j+1 / 2}=\frac{1}{4}\left(\rho_{i+1 / 2, j}+\rho_{i-1 / 2, j}+\rho_{i+1 / 2, j+1}+\rho_{i-1 / 2, j+1}\right)
$$

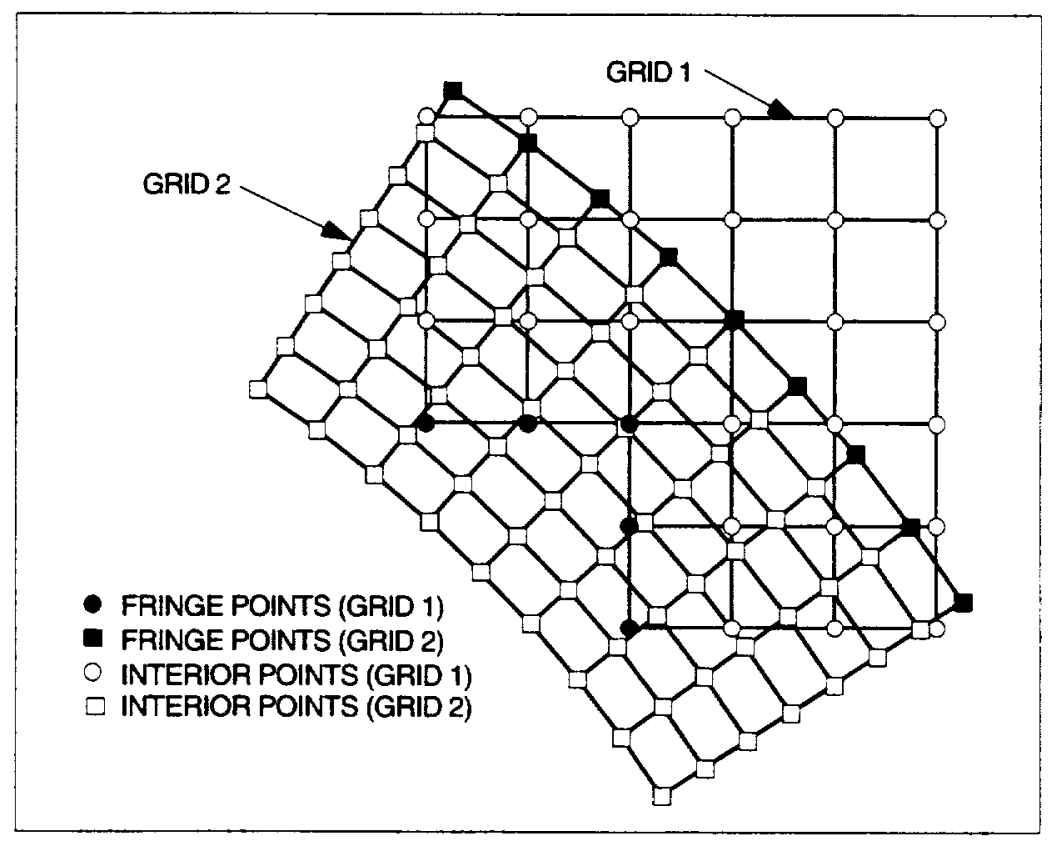

Fig. 1 Schematic of a typical grid-to-grid interface associated with the overset or chimera multi-zone grid scheme. 
At the $\eta$-direction flux that occurs directly between a fringe point and an interior point, values of $\rho_{i-1 / 2, j}$ and $\rho_{i+1 / 2, j}$ are not computable with the standard interior scheme. Instead, the standard formula given by Eq. (17) is implemented, followed by the conditional formula given by

$$
\text { if } I B_{i, j}=0 \text { then } \rho_{i, j+1 / 2}=\frac{1}{2}\left(\rho_{i-1 / 2, j+1}+\rho_{i+1 / 2, j+1}\right)
$$

This is implemented at every grid point and only requires the special information already stored in the $I B_{i, j}$ array. In effect, the second-order four-way average is replaced by a first-order two-way average, but only at fringe points (as well as at points inside hole boundaries which are not used). The standard second-order scheme at interior grid points is not compromised. Additional, schemes for computing $\rho_{i, j+1 / 2}$ at fringe points, that are second-order accurate, are available, but have not been used to date.

\section{NUMERICAL RESULTS}

\section{Problem setup and chimera grid arrangement}

To evaluate the attributes of the chimera approach for solving the full potential equation the flow over a two-dimensional circular cylinder is chosen. The simplicity of this problem allows comparisons between both numerical and analytical solutions and between various numerical solutions computed on both single-zone and multiple-zone chimera grids. In this study both incompressible and shocked compressible cases are considered. Because of the existence of an exact analytic solution for incompressible circular cylinder flow, error can be precisely computed and used to determine detailed characteristics of the present chimera approach.

A schematic of the two-zone chimera grid arrangement used in this study to solve for the flow about the upper half of a two-dimensional circular cylinder is displayed in Fig. 2. The outer grid is Cartesian, and the inner grid is polar. Key parameters for this grid arrangement are defined in Fig. 2 and consist of the circular cylinder radius (R1, which is always equal to 0.5 ), $\S$ the inner-polar-grid outer-boundary radius ( $R 2)$, the hole boundary dimensions ( $X 1$ and $Y 1$, which are always equal to each other), and the outer Cartesian grid dimensions (X2 and $Y 2$, which are also always equal to each other).

A typical grid utilizing the problem setup of Fig. 2 is displayed in Fig. 3. The entire computational domain, including the outer-grid-zone outer boundary, is displayed. $\S \S$ Both the outer Cartesian and inner polar grids are equally spaced. The dimensions for this grid are $65 \times 33$ for the outer zone and $33 \times 13$ for the inner zone. As can be seen by the statistics in Fig. 3 , the chimera boundary cell area ratios range from 2.04 to 1.37 at the inner boundary to 0.97 (a constant) at the outer chimera boundary. In other words, at the outer chimera boundary, grid cells from both grid zones are almost identical in size. A blowup view of this grid showing details of the chimera grid interface is displayed in Fig. 4.

In addition to the two-zone chimera cases computed using the grid topology of Fig. 2, single-grid flow computations using a simple polar-grid topology have also been computed for many of the cases studied herein. These single-zone grids are identical to the inner polar grid shown in Figs. 3 and 4 except that more points are added in the radial direction to allow the outer boundary position to approximately match that of the Cartesian grid in the corresponding two-zone case. The outer boundary position for all incompressible flow cases was about four cylinder diameters away from the

\footnotetext{
$\S$ All lengths in this study, including both the $x$ and $y$ coordinates, are nondimensionalized by the circular cylinder diameter.

$\S \S$ This is a typical computational grid for the incompressible simulations only. The compressible cases used the same topology, but with a stretched computational grid occupying a much larger physical domain.
} 
center of the cylinder for both grid arrangements. Complete grid statistics are given for each computation as they are presented.

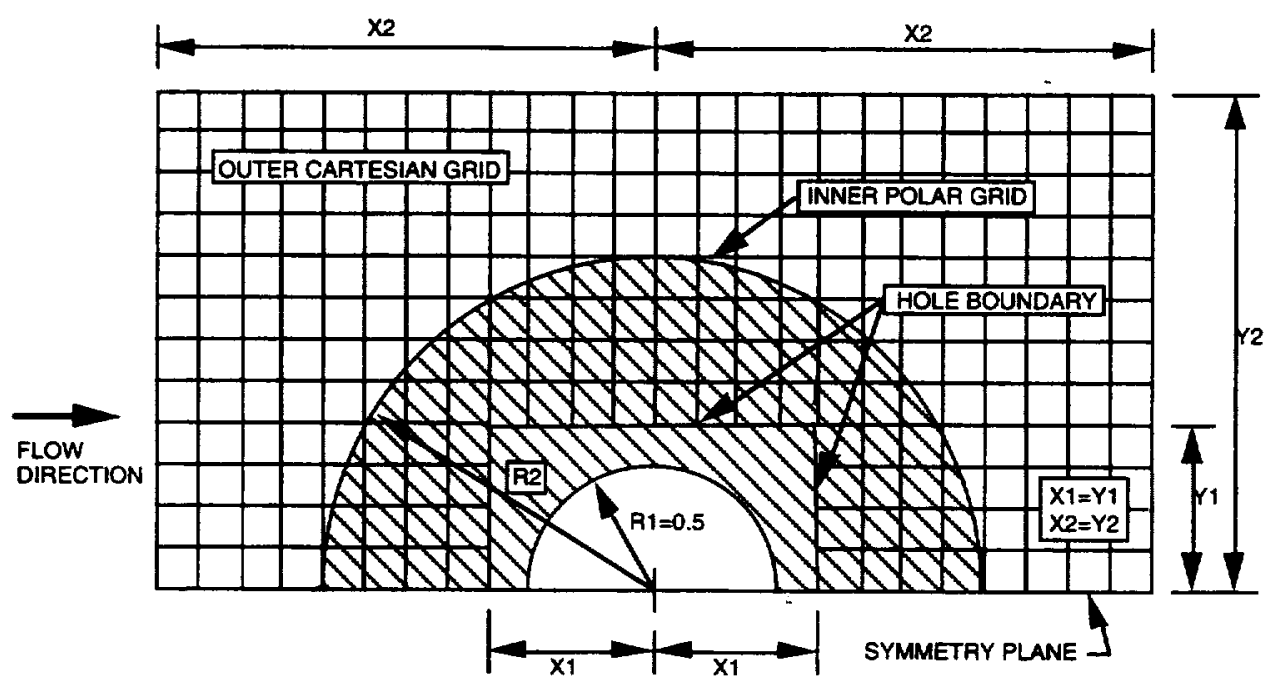

Fig. 2 Schematic of the two-zone chimera grid arrangement used in the present study to solve the flow over the upper half of a two-dimensional circular cylinder.
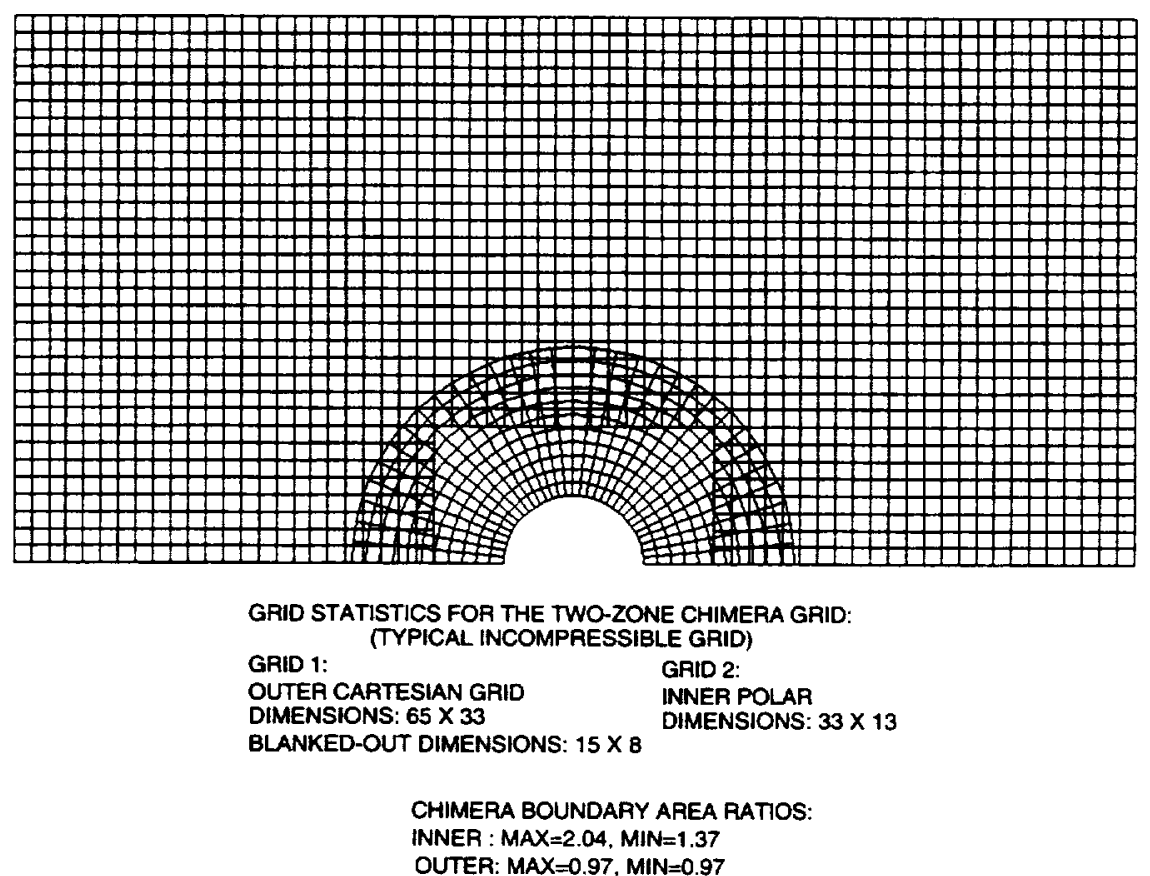

Fig. 3 Typical two-zone chimera grid used in the present study to compute the flow over the upper half of a two-dimensional circular cylinder, incompressible flow cases only, $R 2=1.68, X 1=1.0, X 2=$ 4.0. 


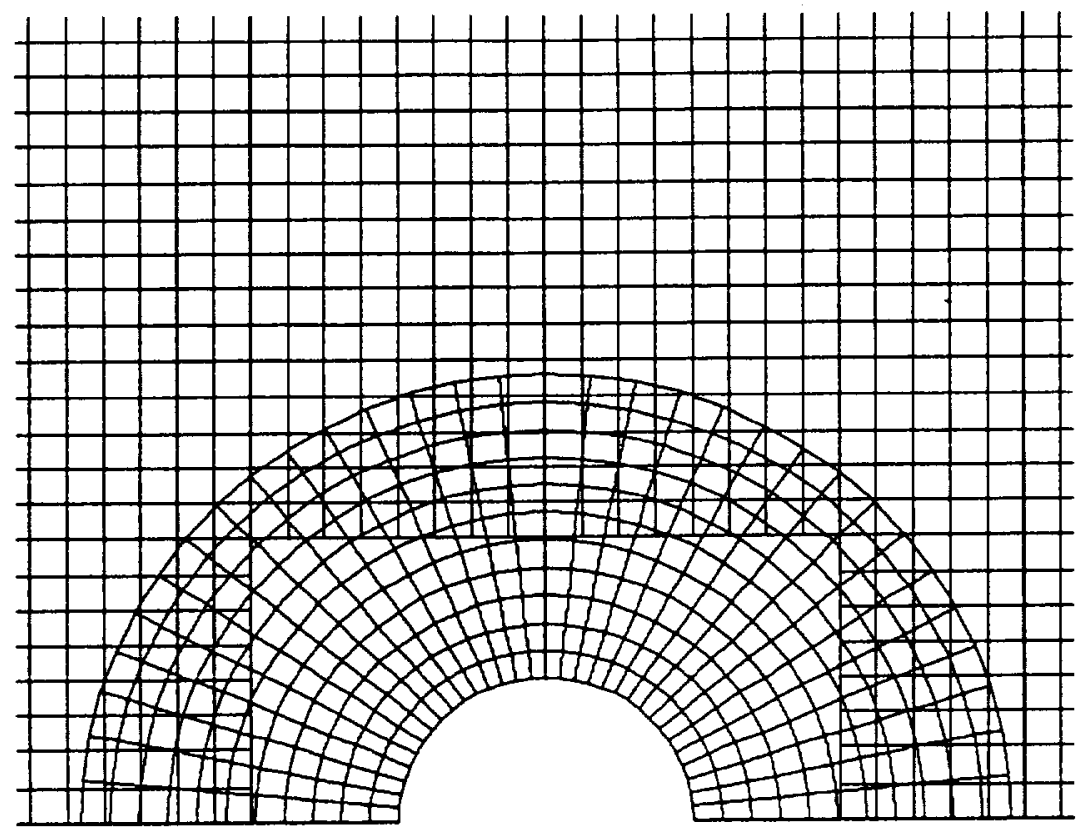

Fig. 4. Blowup of the two-zone chimera grid from Fig. 3 showing detail around the circular cylinder and the chimera overlap region.

All computations presented herein were performed on a Silicon Graphics IRIS Elan 4000 workstation using 64-bit precision. The double precision was required to ensure tight convergence with enough accuracy to be able to compute small errors on fine grids. All computations in which error comparisons were involved were iterated until the maximum residual was reduced by eight orders of magnitude. Typical computational times ranged from a few seconds to a few tens of minutes for most of the cases considered. The convergence rate on the two-grid chimera cases was generally comparable to that of the corresponding single grid cases for most of the solutions performed. One exception to this was the fine-grid, strong-shock compressible case where the chimera-solution convergence rate was about five times slower than the corresponding single-grid convergence rate. Generally, the topics of convergence acceleration, convergence optimization, and code execution efficiency have not been investigated and need more attention.

\section{Incompressible flow}

The first series of results is associated with an incompressible flow solution $\left(M_{\infty}=0\right)$ on a grid twice as fine as the grid displayed in Figs. 3 and 4 , i.e., exactly twice the number of grid cells in each coordinate direction. The outer Cartesian grid contains $129 \times 65$ points, and the inner polar grid contains $65 \times 25$ points. The outer boundary positions for each of the two grid zones and the hole boundary position are both exactly the same as in Figs. 3 and 4 . The cell area ratios along the inner and outer chimera boundaries are also very close to those of Figs. 3 and 4 and range from 0.97 to 1.92. Thus, the truncation error variation due to differing grid resolution from one grid to the next should be relatively small for this case. The single polar grid results computed for comparative purposes for this case are computed on a $65 \times 65$ grid.

Pressure coefficient distributions along the circular cylinder surface and along the downstream stagnation streamline are displayed in Figs. 5 and 6 , respectively. The exact solution is also displayed for comparison. In Fig. 6, pressure coefficient distributions for both the inner and outer grids, including the region of overlap, are displayed. All results are in excellent agreement with each other, both on the cylinder surface and along the downstream stagnation streamline. Additional information about the two-zone chimera solution can be obtained by examining the pressure contours displayed in Figs. 7 and 8 . Contours surrounding the entire circular cylinder are shown in Fig. 7, and a blowup of 
the contours in the aft portion of the circular cylinder are shown in Fig. 8. The actual Mach number used in this computation is 0.01 . This essentially incompressible Mach number is used instead of zero to allow a very slight density variation that is picked up by the PLOT3D graphics program 33 to produce pressure contours. Contours are plotted for both grid zones which creates two lines for each contour level in the chimera overlap region. Close examination of the pressure contours in the overlap region show remarkable continuity, both on slope and position, with only small variations present.

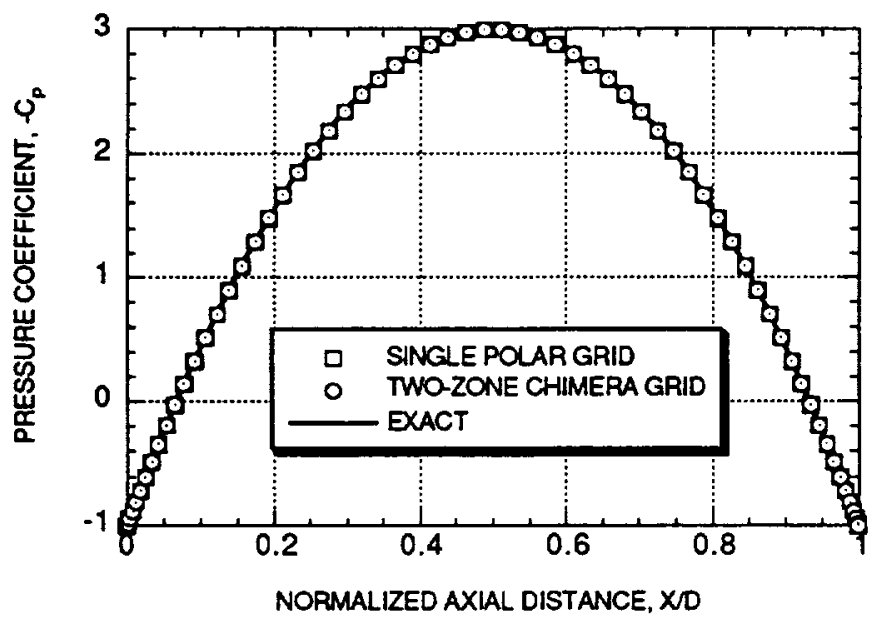

Fig. 5 Surface pressure coefficient distributions comparing results from the single-zone and twozone numerical approaches with an exact analytical solution; $M_{-}=0$; single-zone grid: $65 \times 65, \mathrm{R} 2=$ 3.65 ; two-zone grid: inner $=65 \times 25$, outer $=129 \times 65, \mathrm{R} 2=1.68, \mathrm{X} 1=1.0, \mathrm{X} 2=4.0$.

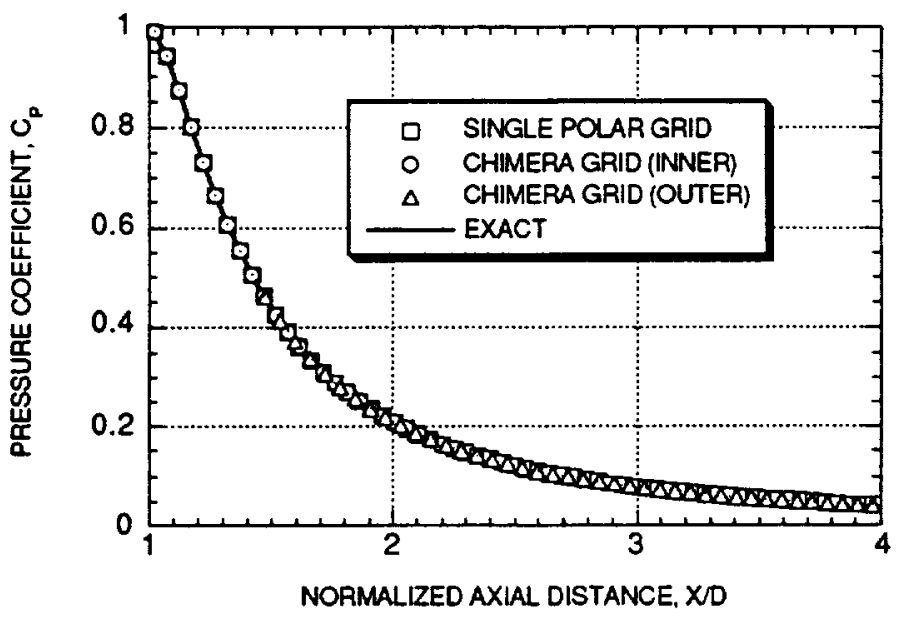

Fig. 6 Pressure coefficient distributions along the downstream stagnation streamline comparing results from the single-zone and two-zone numerical approaches with an exact analytical solution; $M_{\infty}=0$; single-zone grid: $65 \times 65, \mathrm{R} 2=3.65$; two-zone grid: inner $=65 \times 25$, outer $=129 \times 65, \mathrm{R} 2=$ $1.68, X_{1}=1.0, X_{2}=4.0$. 


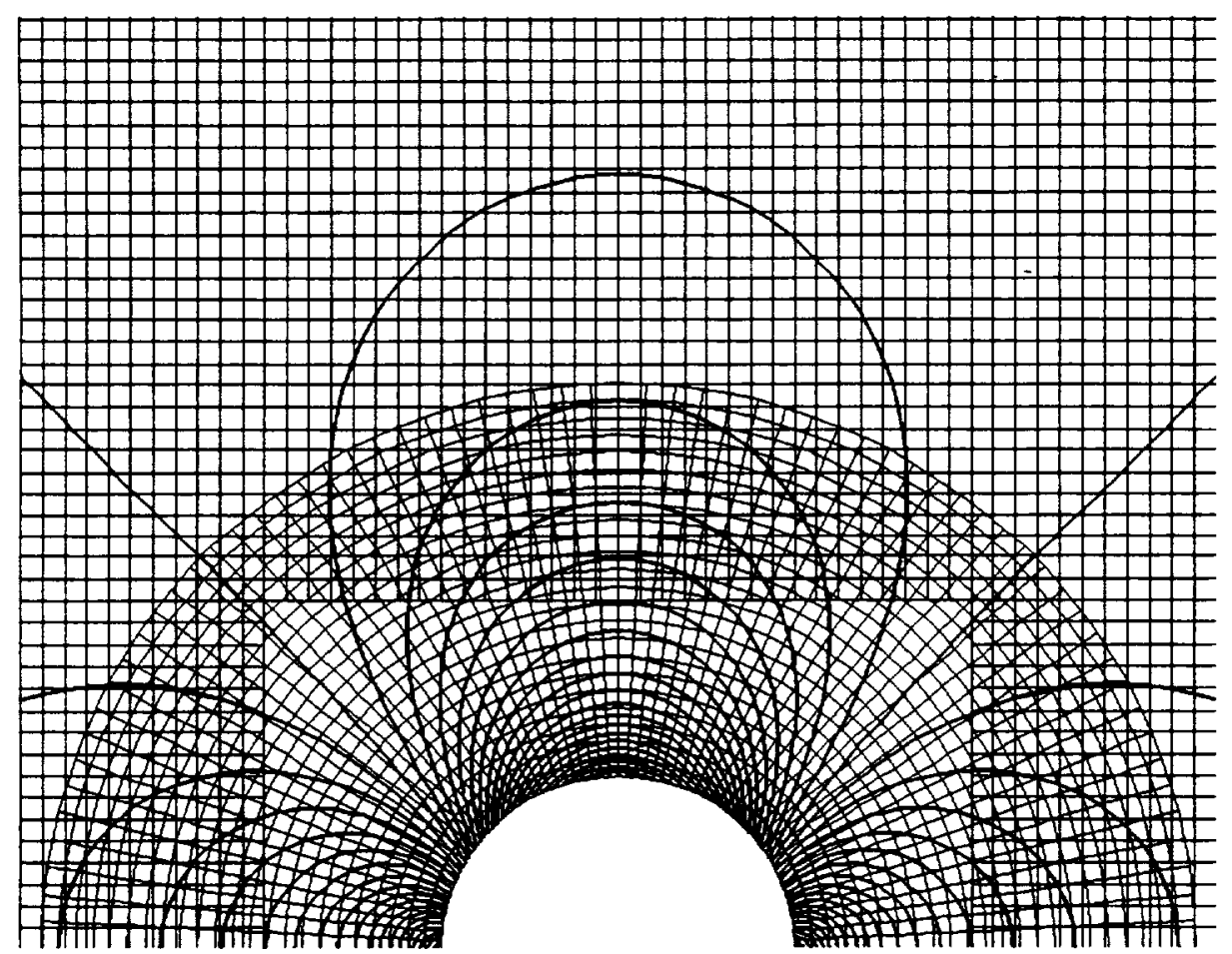

Fig. 7 Pressure contours surrounding the circular cylinder showing the inner polar grid solution, a portion of the outer Cartesian grid solution, and the region where the two solutions overlap, $M_{\infty}=0.01$, two-zone grid: inner $=65 \times 25$, outer $=129 \times 65, \mathrm{R} 2=1.68, \mathrm{X} 1=1.0, \mathrm{X} 2=4.0$.

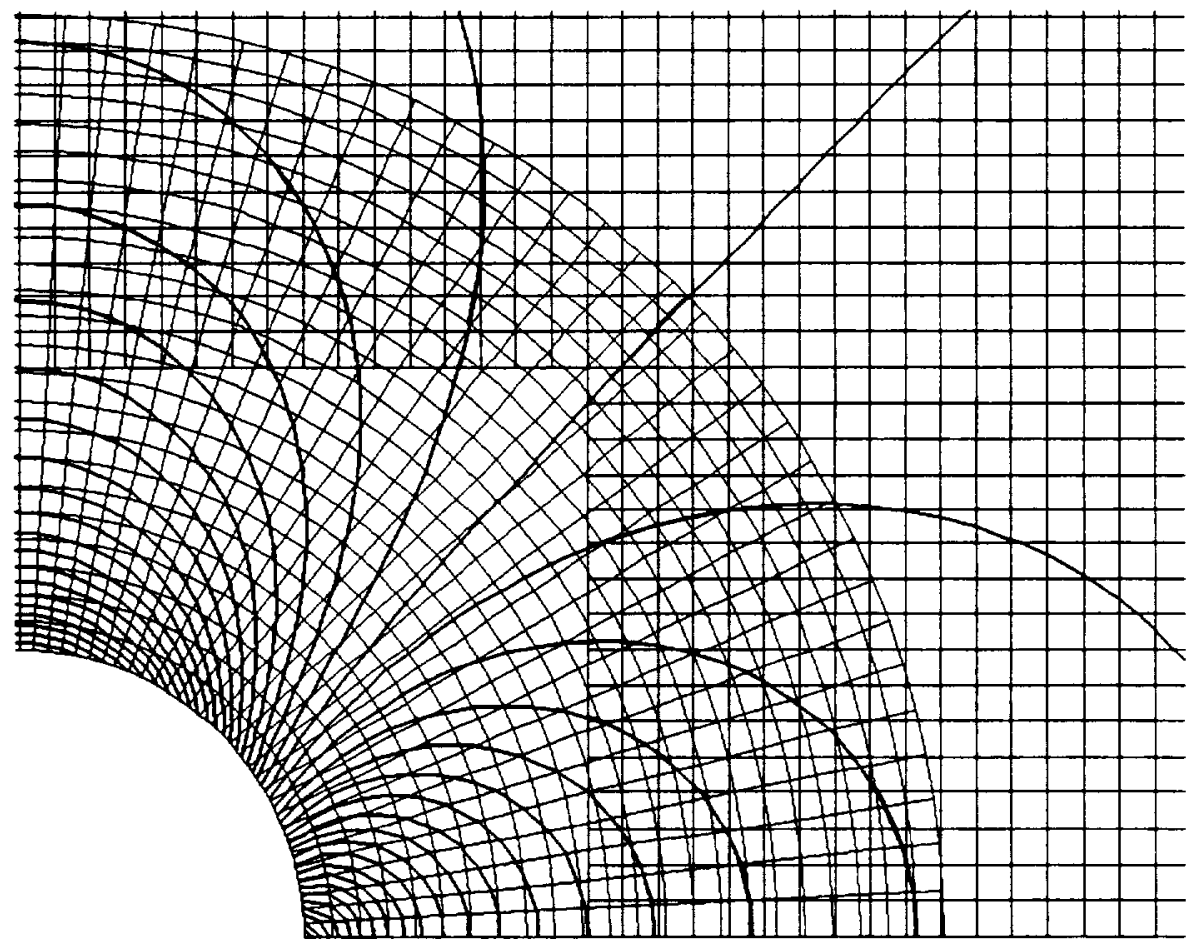

Fig. 8 Blowup of the pressure contours from Fig. 7 showing the chimera interface region in the aft portion of the flow field, $M_{\infty}=0.01$. 
Because the exact solution for the flow about a circular cylinder immersed in an incompressible uniform freestream is available, additional insight into the level of accuracy of the present computations can be made by examining error. Figures 9 and 10 show the error in pressure coefficient along the circular cylinder surface and along the downstream stagnation streamline, respectively. Results for both the two-grid chimera and single polar grid cases are included. The error in surface pressure coefficient (Fig. 9) is quite smooth, and like the pressure itself, is sinusoidal in shape. The two numerical results are not identical but are remarkably similar. The peak error (just above $0.3 \%$ ) occurs at the circular cylinder maximum thickness where the maximum flow acceleration occurs. The minimum error is zero and occurs at each of the two stagnation points, which are exactly enforced via boundary condition.

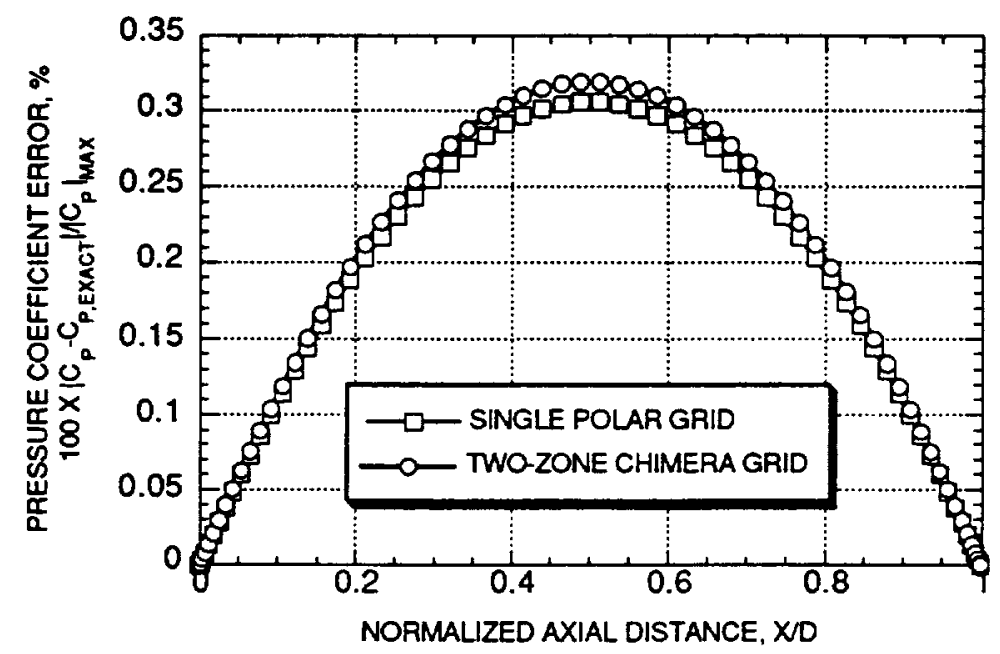

Fig. 9 Surface pressure coefficient error distributions comparing results from the single-zone and two-zone numerical approaches; $M_{\infty}=0$; single-zone grid: $65 \times 65, \mathrm{R} 2=3.65$; two-zone grid: inner $=$ $65 \times 25$, outer $=129 \times 65, R 2=1.68, \times 1=1.0, \times 2=4.0$.

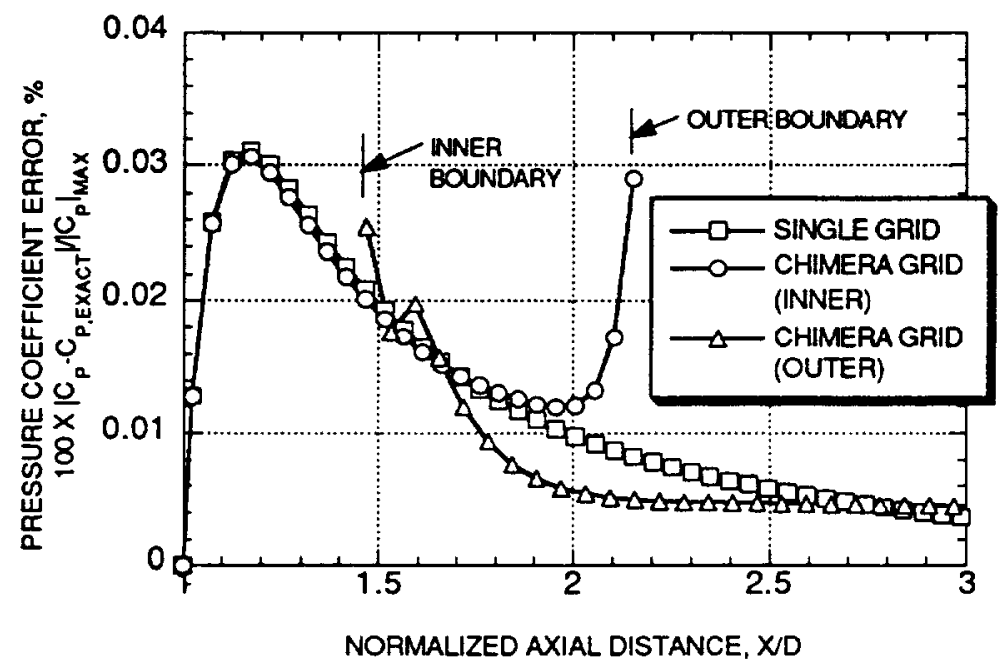

Fig. 10 Pressure coefficient error distributions along the downstream stagnation streamline comparing results from the single-zone and two-zone numerical approaches; $M_{\infty}=0$; single-zone grid: $65 \times 65, \mathrm{R} 2=3.65$; two-zone grid: inner $=65 \times 25$, outer $=129 \times 65, R 2=1.68, X 1=1.0, \times 2=4.0$. 
In Fig. 10, the pressure coefficient error along the downstream stagnation streamline starts at zero (the downstream stagnation point) and then rapidly grows to a local maximum (about $0.03 \%$ ) just five grid points downstream of the stagnation point. The pressure coefficient error then drops rapidly. This behavior is shared by both the two-grid chimera and the single polar-grid solutions, until the chimera interface boundaries are encountered. The pressure coefficient error for the two-grid chimera solution in the vicinity of the chimera interface boundaries is not well behaved. This is especially true for the inner-grid solution at the outer chimera interface and the outer-grid solution at the inner interface. This behavior is typical of the solutions computed in this study and is caused by the error aggravating numerical differentiation process, which is required to get pressure from the velocity potential. That is, the interpolation error in the velocity potential generated by the chimera approach at the two chimera interface boundaries is effectively magnified by numerical differentiation and produces local maxima in the pressure error in the vicinity of the interpolation boundaries. In addition, this behavior is magnified even more as the outer grid is coarsened (a likely scenario in many applications). In the present case the outer chimera boundary area ratio is approximately unity. If the outer grid were coarsened by a factor of two in each direction, the truncation error on the outer grid for the present second-order scheme would increase by a factor of four. The pressure error on the inner grid at the interface boundary would also dramatically increase.

The effect of the error aggravating numerical differentiation process can be further understood by examining the error in the velocity potential itself. Just such a plot for the velocity potential error along the downstream stagnation streamline is displayed in Fig. 11 for the identical computation that produced Figs. 9 and 10 . In Fig. 11 it is seen that the velocity potential error has a local maximum at the downstream stagnation point and then exponentially decays further downstream. This behavior, with minor variations, is displayed by both the two-grid chimera solution and the single polar-grid solution. Velocity potential error through the interface boundary is generally well below $0.01 \%$ and very smooth, whereas the pressure coefficient error in the same area (from Fig. 10) is about an order of magnitude larger and rather poorly behaved.

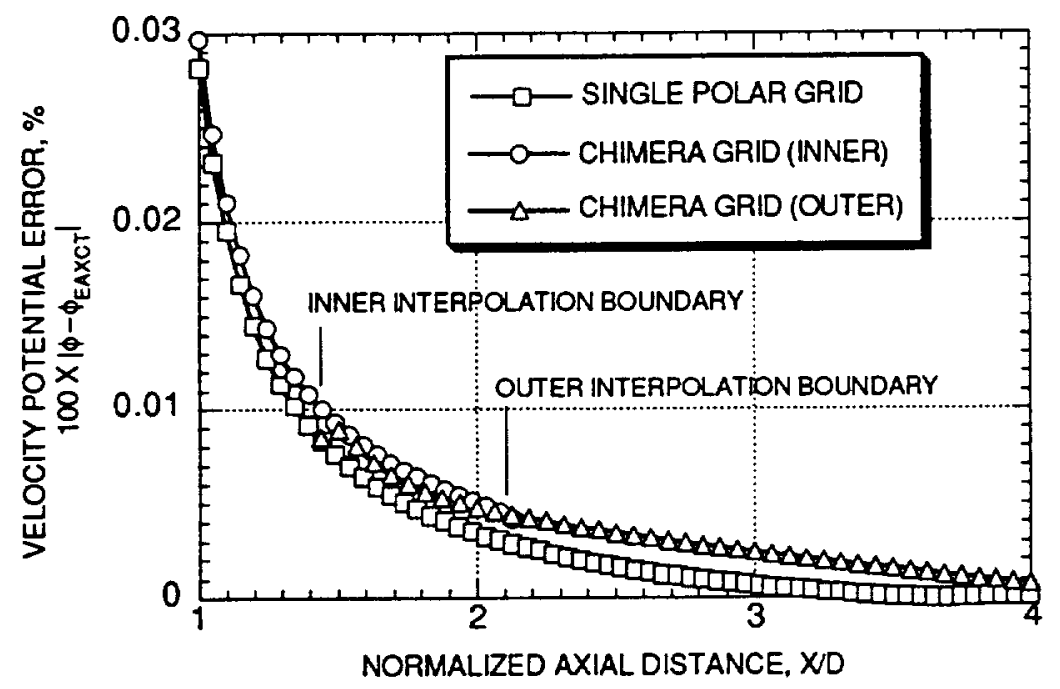

Fig. 11. Velocity potential error distributions along the downstream stagnation streamline comparing results from the single-zone and two-zone numerical approaches; $M_{\infty}=0 ;$ single-zone grid: $65 \times 65$, $R 2=3.65$; two-zone grid: inner $=65 \times 25$, outer $=129 \times 65, R 2=1.68, \times 1=1.0, \times 2=4.0$.

At first thought, comments from the above two paragraphs may seem to be alarming, since in aerodynamic applications the pressure is a much more important quantity than the velocity potential. However, the situation is actually quite good for the present chimera approach for several reasons. First of all, the effect of the chimera interface boundary dies out rapidly with distance. The seemingly large pressure coefficient error at the chimera interface in Fig. 10 has little affect on the error at the cylinder surface (and of course, it's really the surface pressure that is most often the point of interest 
in aerodynamics). Second, the pressure coefficient errors displayed across the chimera interface in Fig. 10 are really quite small. They are not even visible in Fig. 5 , which is a plot of the actual pressure in the same location. Lastly, the pressure coefficient error at the interface boundaries of Fig. 10 are an order of magnitude smaller than the peak pressure coefficient error over the entire solution. Incidentally, the peak pressure coefficient error for the present solution is shared by both the chimera and single-zone solutions and occurs at the maximum thickness of the circular cylinder (see Fig. 9). The above results are interesting and help shed light on how and where chimera interpolation error affects a solution, but the most fundamental issue (for any numerical scheme) is how the error is reduced as the grid is refined. Results from just such a grid refinement study are presented in the next section.

\section{Grid refinement study}

A grid refinement study is now presented using the two-dimensional circular cylinder geometry and grid arrangement discussed above. Pertinent statistics for the grids utilized in the grid refinement study are presented in Table 1 for the two-zone chimera approach and in Table 2 for the single-zone polar-grid approach. In this series of computations (for the chimera approach) the outer Cartesian grid is a factor of four coarser (in terms of area ratio) than the inner polar grid at the outer chimera boundary interface. This situation is easily seen in Table 1 by looking at the outer boundary area ratios, which are still constant, but are now a factor of four (approximately) larger than the area ratio used for the computational results presented in Figs. 3-11. A coarser outer Cartesian grid is chosen for the grid refinement study because it represents a more difficult case to demonstrate grid independence. Truncation error from the outer grid at the chimera interface will be a factor of four larger and will create a more difficult situation for the velocity potential interpolation. A coarser outer Cartesian grid was also chosen because it is more likely to arise in practical application computations.

Table 1. Summary of grid statistics for the two-zone chimera grid refinement study, $R 2=1.68$,

\begin{tabular}{|c|c|c|c|c|c|c|c|}
\hline \multirow{3}{*}{0.58} & \multicolumn{3}{|c|}{ Grid omensions: } & \multicolumn{4}{|c|}{ Wrimera thiterface area ratios } \\
\hline & & & & oute & & 1mine & dary. \\
\hline & outtes & Shaners & Glankeg & $\max$ & $\min$ & $\max$ & Milin \\
\hline $\begin{array}{c}2 G 17 \\
2 G 33 \\
2 G 65 \\
2 G 129 \\
2 G 257\end{array}$ & $\begin{array}{c}17 \times 9 \\
33 \times 17 \\
65 \times 33 \\
129 \times 65 \\
257 \times 129\end{array}$ & $\begin{array}{c}17 \times 7 \\
33 \times 13 \\
65 \times 25 \\
129 \times 49 \\
257 \times 97 \\
\end{array}$ & $\begin{array}{c}3 \times 2 \\
7 \times 4 \\
15 \times 8 \\
31 \times 16 \\
63 \times 32 \\
\end{array}$ & $\begin{array}{l}3.89 \\
3.87 \\
3.87 \\
3.86 \\
3.86\end{array}$ & $\begin{array}{l}3.89 \\
3.87 \\
3.87 \\
3.86 \\
3.86 \\
\end{array}$ & $\begin{array}{l}13.05 \\
9.33 \\
7.69 \\
7.07 \\
6.71 \\
\end{array}$ & $\begin{array}{l}9.37 \\
6.55 \\
5.25 \\
4.95 \\
4.77 \\
\end{array}$ \\
\hline
\end{tabular}

Table 2. Summary of grid statistics for the single-zone grid refinement study involving the polar grid topology, $\mathrm{R} 2=3.65$.

\begin{tabular}{|c|c|}
\hline Casee & Giddimensions \\
\hline $1 \mathrm{G} 17$ & $17 \times 17$ \\
$1 \mathrm{G} 33$ & $33 \times 33$ \\
$1 \mathrm{G} 65$ & $65 \times 65$ \\
$1 \mathrm{G} 129$ & 129129 \\
$1 \mathrm{G} 257$ & $257 \times 257$ \\
\hline
\end{tabular}

Each of the five grids described in Table 1 was carefully constructed so as to have the same outer boundaries, the same hole boundaries, and the same amount of spatial overlap at the chimera interface. The grid generation process (for both the two-zone and single-zone cases) was to first generate the finest grid (i.e., $2 \mathrm{G} 257$ or 1G257) and then (if a coarser grid was required) retain only every 2 nd, 4 th, 8 th, or 16 th point in each direction. This process worked well to ensure proper accuracy during the grid refinement study. 
The first results of the grid refinement study are displayed in Figs. 12 and 13 . Figure 12 shows the global rms error in the velocity potential plotted against grid spacing for each of the grids described in Tables 1 and 2. Velocity potential values at all grid points, including all values in the chimera overlap region for the two-grid chimera cases, are used in computing this error. As can be seen from Fig. 12 the velocity potential error curves for both the two-grid chimera and single-grid approaches are almost identical. Both drop at the expected second-order rate. Figure 13 shows the same chimeraapproach error curve as in Fig. 12 along with curves from each of the two chimera component grids. As expected the velocity potential error is much larger on the inner grid where the largest solution gradients exist. All curves approach the expected second-order slope as the grid is refined.

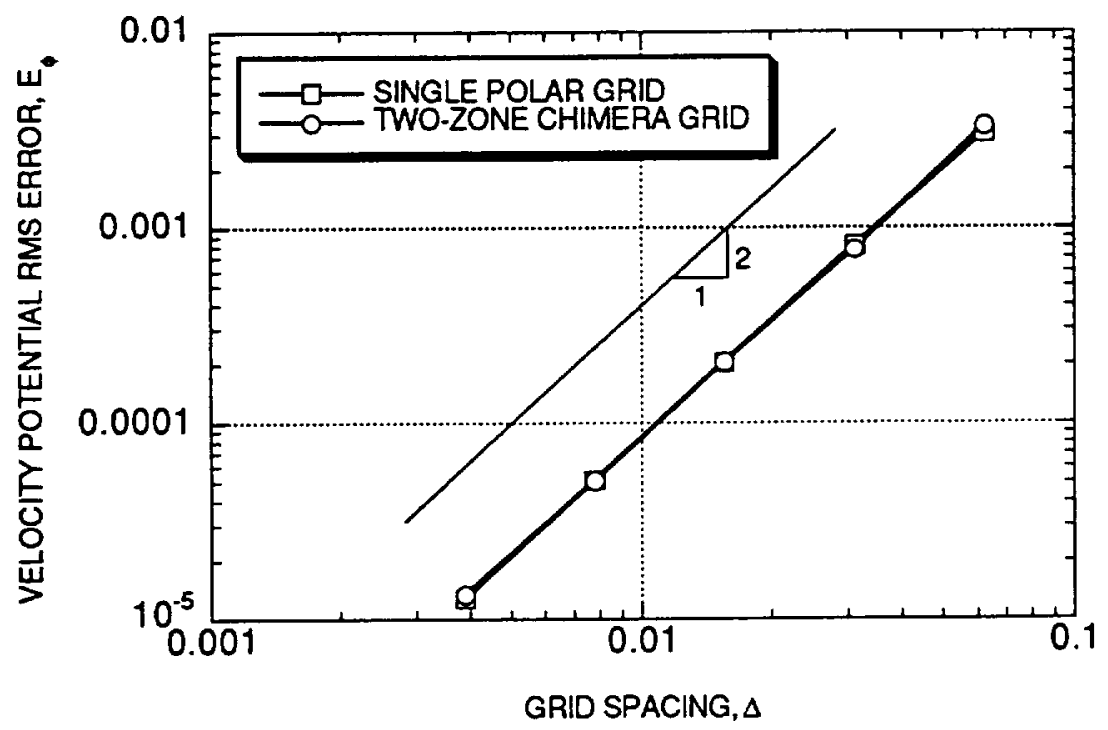

Fig. 12 Effect of grid refinement on the global rms error in the velocity potential; $M_{\infty}=0$; single-zone grid: $R 2=3.65$; two-zone grid: $R 2=1.68, X 1=1.0, X 2=4.0$.

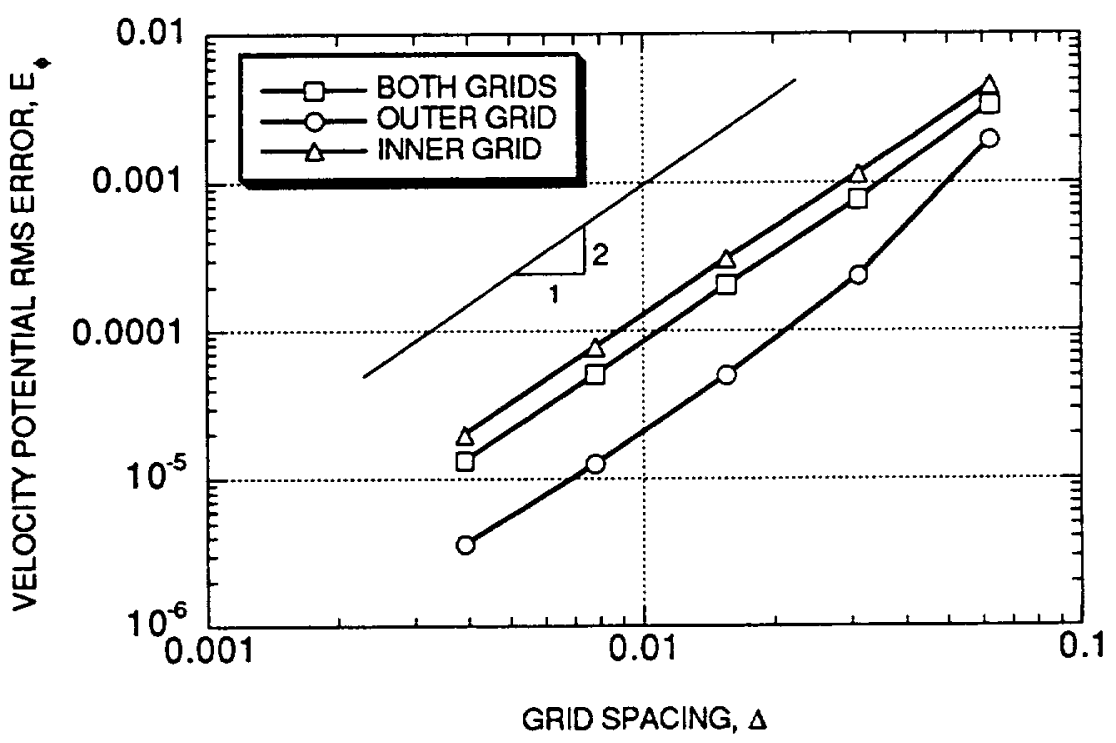

Fig. 13 Effect of grid refinement on the global $\mathrm{rms}$ error in the velocity potential (individual results for each grid zone in the two-zone chimera case), $M_{\infty}=0, \mathrm{R} 2=1.68, \mathrm{X} 1=1.0, \mathrm{X} 2=4.0$. 
Another important aspect to explore in a grid refinement study (especially for aerodynamic applications) is the behavior of the solution at the aerodynamic surface of interest. Figure 14 shows the rms error in the surface pressure coefficient plotted against grid spacing for each of the grids described in Tables 1 and 2. Again, both curves are nearly identical dropping at the expected second-order rate. Another way to display grid refinement effects on the surface pressure error is as shown in Fig. 15. In this figure, surface pressure error is plotted on a logarithmic scale against axial distance for the four finest grids in Tables 1 and 2. Each set of two curves representing computations that have the same circumfrential grid spacing are in good agreement. The drop in error for a grid space halving is almost exactly four across the entire curve no matter which two levels of grid refinement are used. (Incidentally, the coarsest grid results were omitted from this plot because of a plotting utility limitation on the number of curves that could be plotted at one time.) In summary, the interpolation-induced error at the chimera interface does not reduce the chimera scheme's ability to compute accurate numerical solutions to the full potential equation relative to a single-grid approach. This important result is obtained for both a global measure of error based on the rms error in the velocity potential and an important local measure of error based on the rms error in the surface pressure.

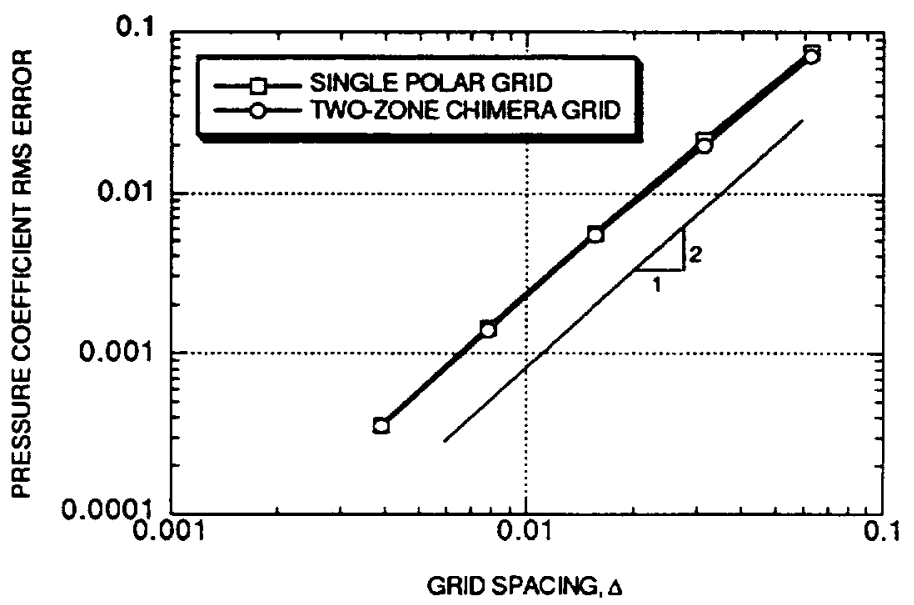

Fig. 14. Effect of grid refinement on the rms error in the surface pressure coefficient, $M_{\infty}=0, R 2=$ 1.68, $\mathrm{X} 1=1.0, \mathrm{X} 2=4.0$.

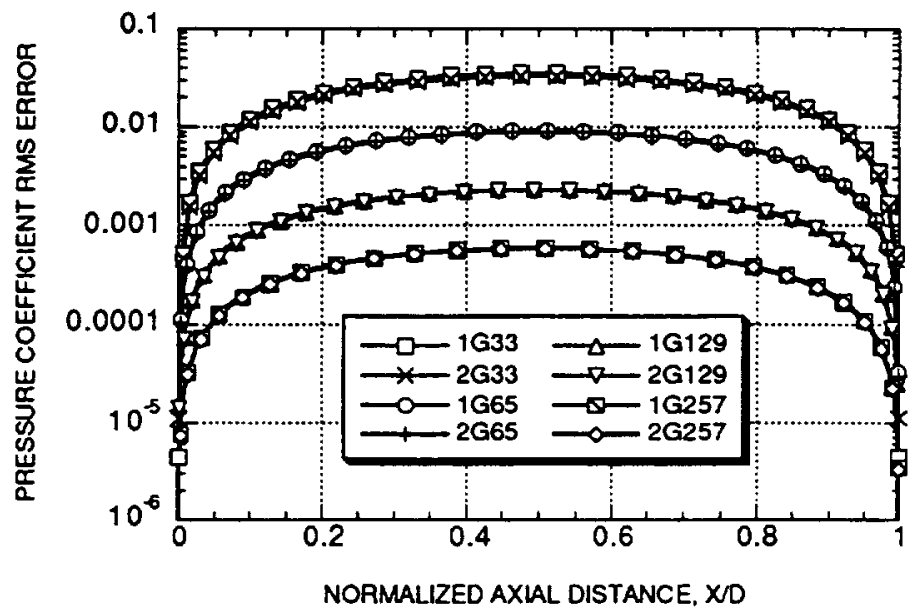

Fig. 15. Effect of grid refinement on the spatial distribution of the surface pressure coefficient error. For clarity a fixed number of points (about 30 ) has been plotted for each curve; $M_{\infty}=0$; single-zone grid: $R 2=3.65$; two-zone grid: $R 2=1.68, X 1=1.0, X 2=4.0$. 


\section{Compressible flow}

The next case used to evaluate the chimera approach is a compressible flow case $\left(M_{\infty}=0.5\right)$ containing a strong shock wave. The maximum value of the local normal shock Mach number, which is located on the circular cylinder surface just upstream of the shock wave, is about 2.2.\$ The geometry and general grid topology for the compressible cases are identical to the previous incompressible cases. However, one change was to move the outer Cartesian grid hole boundaries as close to the circular cylinder geometry as possible. This allows an evaluation of the chimera interpolation scheme as the shock wave passes through the inner interpolation boundary. The new hole boundary position allows a minimum clearance of two computational cells between the hole boundary and the circular cylinder geometry.

Results for the compressible case are displayed in Figs. 16-20. The grid used for this case consists of a $129 \times 49$ inner polar grid and a $257 \times 129$ outer Cartesian grid. A corresponding single-zone computation was also performed on a $129 \times 129$ polar grid for comparative purposes. For both the two-zone and single-zone grids, a small amount of stretching is employed to move the outer boundary to a position about 17 diameters away from the circular cylinder where freestream conditions are implemented. The grids used in this case are exactly twice as fine in each coordinate direction as those used in the incompressible case presented in Figs. 5-11. Because the hole boundary was moved in toward the circular cylinder where the polar grid is finer, the grid cell area ratios along the inner interface boundary are slightly larger than for the incompressible case. In the present case the cell area ratios range from about 1.8 to 3.0 along the inner interface boundary. This can be verified by close examination of the interface boundary that is displayed in Figs. 16-17.

Mach number contours for the two-zone chimera computation are displayed in Fig. 16. The entire circular cylinder geometry and the entire inner interface boundary are displayed in this figure. A blowup of the Mach number contours in the vicinity of the shock wave is displayed in Fig. 17. As can be seen from these figures, the Mach number contours pass through the chimera interface boundary smoothly and without discontinuities in slope. Away from the shock and in the overlap region, contours from the inner and outer grids are nearly coincident. In the vicinity of the shock it is evident that the coarseness associated with the outer grid relative to the inner grid (especially in the axial direction) causes a slight additional smearing in the shock wave at the interface boundary. This is an expected behavior as the higher gradient associated with the shock wave cannot completely be supported on the coarser outer grid. For comparative purposes, Figs. 18 and 19 display Mach number contour plots for a single-grid solution using identical plotting parameters as those of Figs. 16 and 17, respectively. Careful comparison of these figures shows that the single-zone polar grid and the inner-chimera polar grid solutions are identical in every respect including detail in the supersonic region and around the shock.

\$ The shock wave that results for this case far exceeds the full potential formulation's ability to produce accurate results. In fact, one manifestation of error for this solution is the prediction of a much stronger shock with a higher upstream Mach number than that predicted by the Euler equations. In addition, viscous effects, which are currently being neglected, will have a significant influence on this flow field. As such, results presented for this case are not physically correct. But, it is important to note that these errors are associated with the full potential formulation and not the present numerical scheme. The present case represents an interesting and difficult numerical test for the present full potential chimera scheme, a test that is more difficult than what is likely to occur in any practical application. Thus, the primary emphasis for this series of calculations will be numerical-tonumerical comparisons between single-grid and multiple-grid computations. 


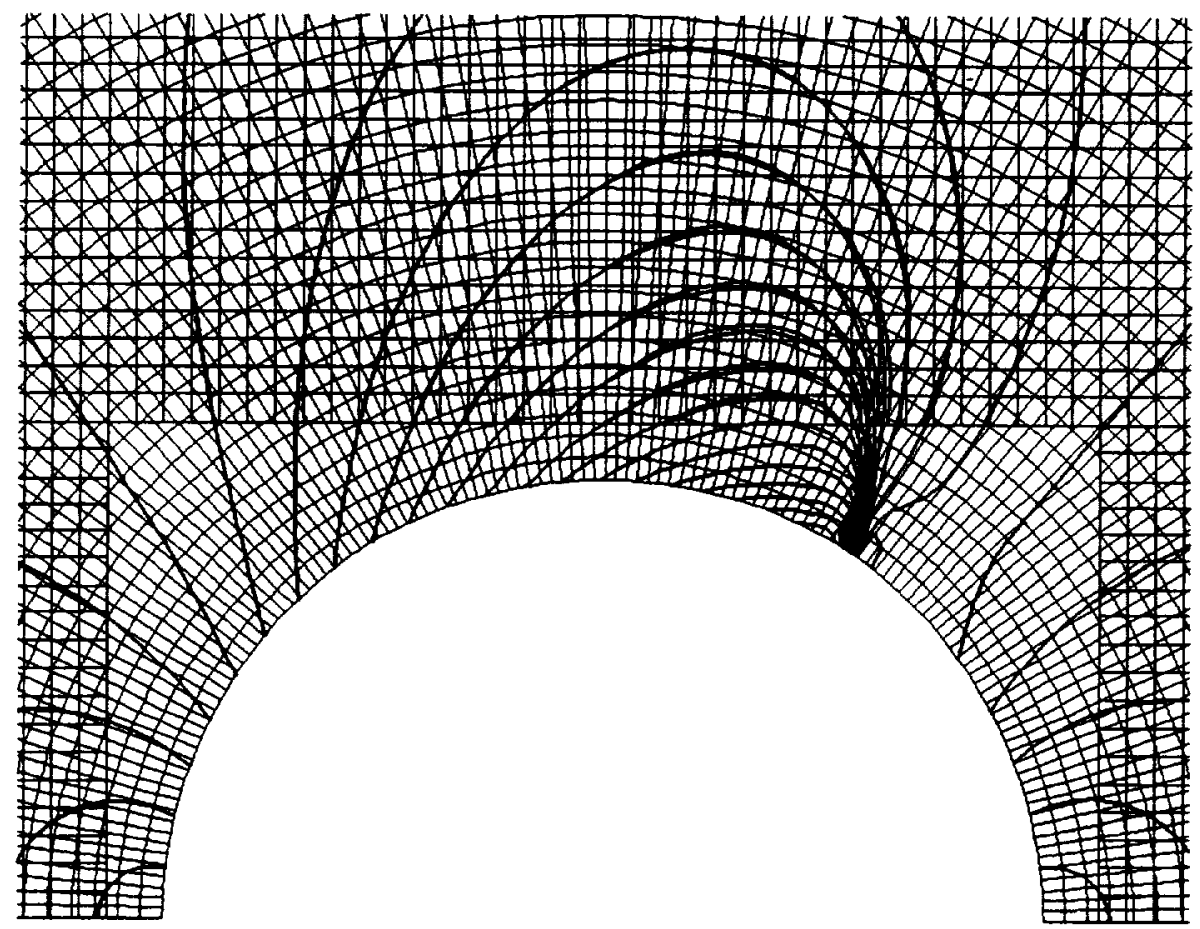

Fig. 16 Mach number contours surrounding the circular cylinder computed using the two-zone chimera grid. Details displayed include the entire surface solution and the entire inner chimera interface boundary, $M_{\infty}=0.5$, inner grid $=129 \times 49$, outer gird $=257 \times 129, R 2=2.46, X 1=0.5625$, $\mathrm{X} 2=17.5$.

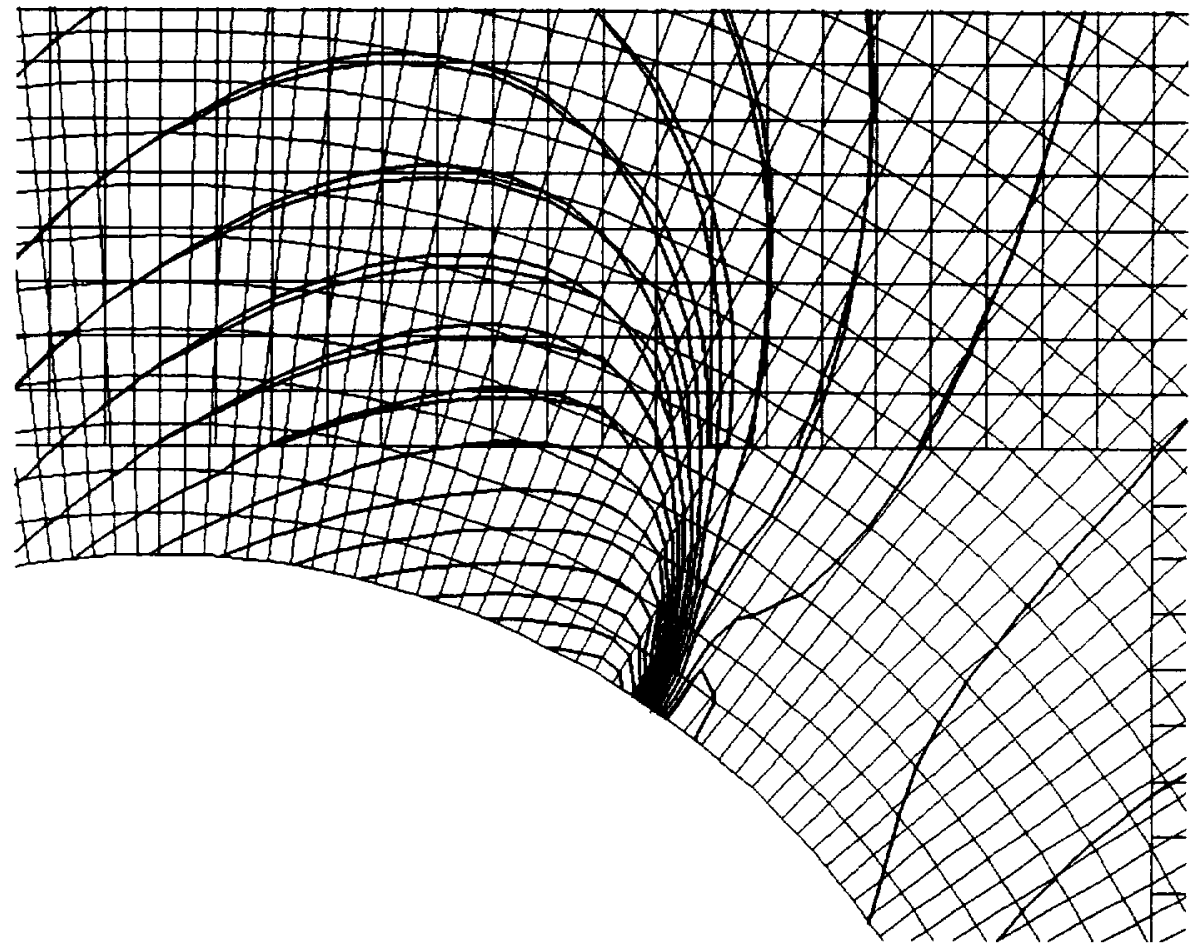

Fig. 17 Blowup of the Mach number contour plot of Fig. 16 showing details around the shock wave; $M_{\infty}=0.5$. 


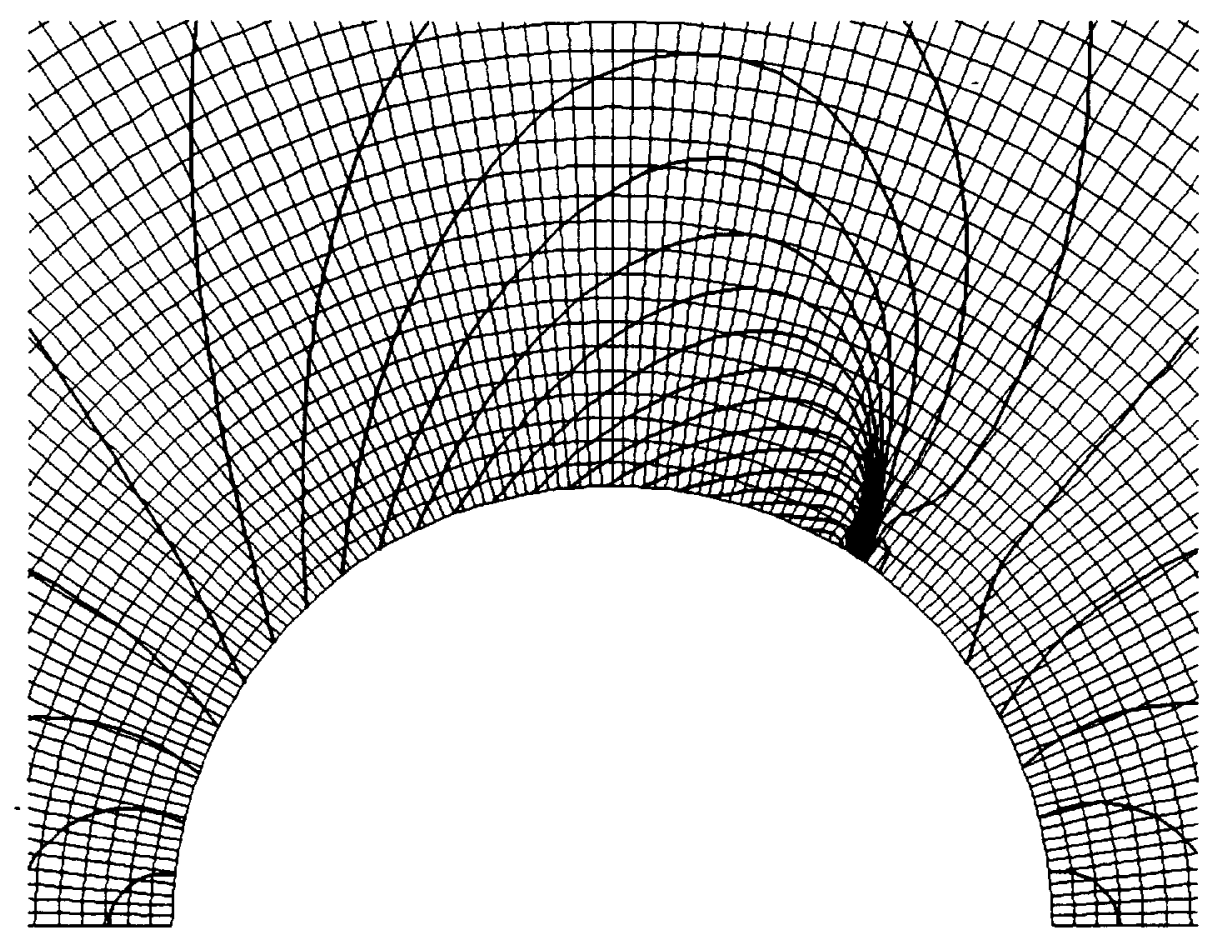

Fig. 18 Mach number contours surrounding the circular cylinder computed using the one-zone polar grid. Plotting area and contour levels are identical to Fig. $16, M_{\infty}=0.5$, grid $=129 \times 129, R 2=18.1$.

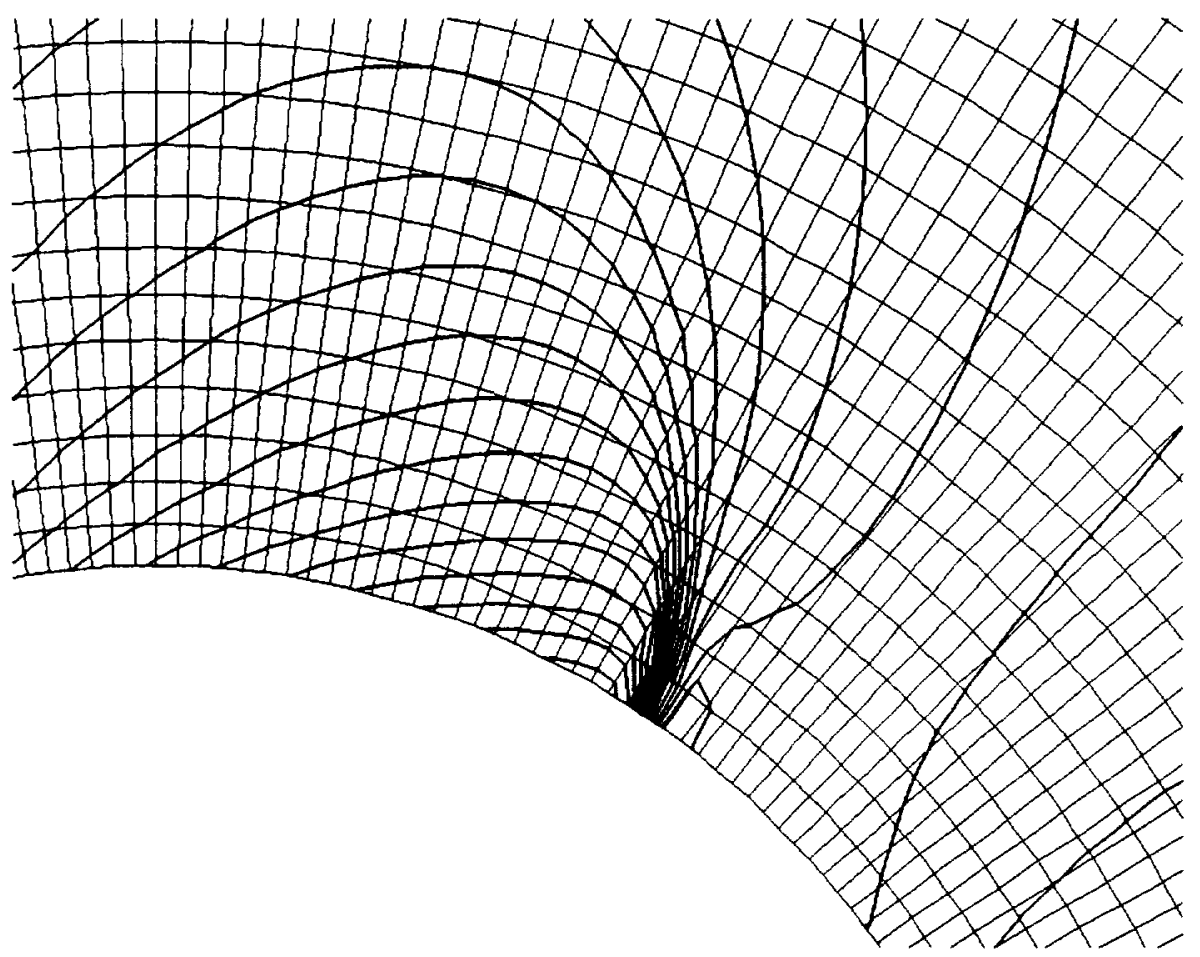

Fig. 19 Blowup of the Mach number contour plot of Fig. 18 showing details around the shock wave. Plotting area and contour levels are identical to Fig. $17, M_{\infty}=0.5$. 
Details associated with the surface solution are compared in Fig. 20 where surface pressure coefficient distributions are displayed for the two-zone and single-zone solutions. As can be seen from this figure the two results are virtually identical. Both results produce an essentially one-grid-cell shock at about $X / D=0.77 . \S$ To enhance the sharpness of the shock obtained in the computation of Figs. 16-20, a value of 0.6 was utilized for the parameter $C$ from Eq. (10).

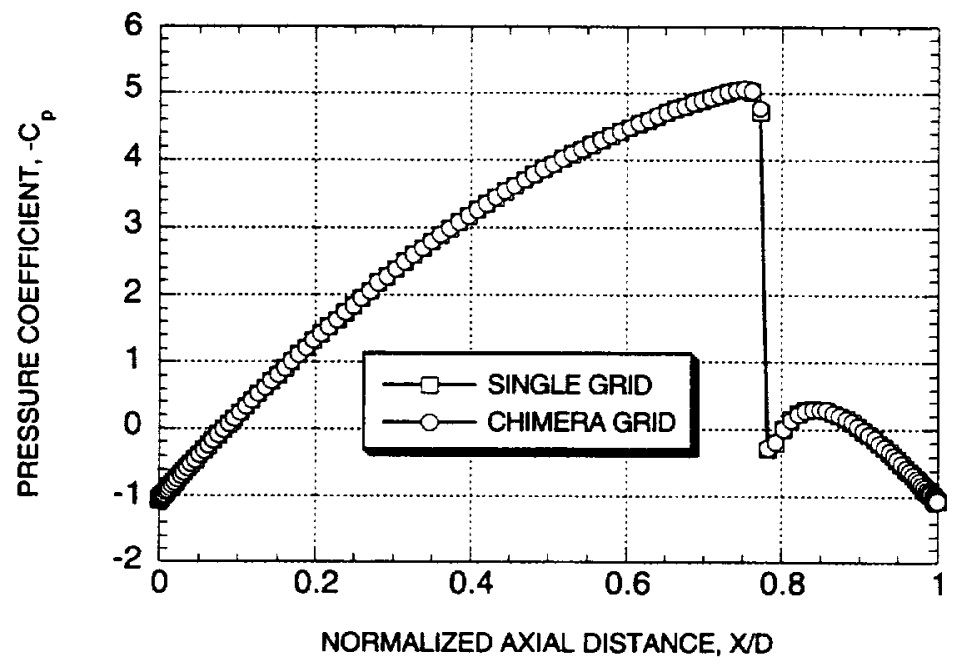

Fig. 20 Surface pressure coefficient distributions comparing the single-zone polar and two-zone chimera grid solutions; $M_{\infty}=0.5$; two-zone grid: inner $=129 \times 49$, outer $=257 \times 129, R 2=2.46, \mathrm{X} 1=$ $0.5625, \mathrm{X} 2=17.5$; single-zone grid $=129 \times 129, \mathrm{R} 2=18.1$.

\section{Outer grid refinement study}

The effect of grid refinement on the present full potential numerical scheme utilizing both single-zone and two-zone approaches was studied in a previous section using the incompressible circular cylinder solution. Now it is of interest to study the effect of grid refinement when only the outer grid is refined. The shocked compressible solution of the last section is used for this study. In this series of cases the inner grid dimensions are fixed at 129X49 and the outer grid dimensions are varied from 17X9 to 257X129. A summary of these grids with additional statistics is given in Table 3 . For this series of cases the hole boundary location has been returned to the location used for the incompressible grid refinement study. This is required to allow enough clearance for the chimera interpolation procedure when the coarsest outer grid is utilized.

Results from the outer grid refinement study are displayed in Figs. 21 and 22 . Figure 21 shows a comparison of surface pressure coefficient distributions for a two-dimensional circular cylinder flow field computed using the sequence of grids listed in Table 3 . One additional curve corresponding to no outer grid is displayed in this figure, i.e., the inner grid of 129X49 points with freestream boundary conditions imposed at its outer boundary, is used to produce this last curve (curve $F$ ). For this case

$\S$ This shock is actually sharper than the shock displayed in the contour plots of Figs. 16-19, because the computational stencil used to compute pressure from the velocity potential for Fig. 20 involved only one streamwise cell, and the computational stencil used to compute the Mach number for the contour plots of Figs. 16-19 used two streamwise cells. The former produces values at half-points in the $i$-direction and the latter produces values at integer points. To ensure comparisons up to the hole boundary for each solution including solutions on grids used in the grid refinement study, values are required at integer points in the finite-difference mesh. 
the outer boundary is located a little less than two diameters beyond the circular cylinder surface. As seen from Fig. 21 the outer grid refinement level has only a small effect on the circular cylinder surface pressure, an amazingly small effect. The results of Fig. 21 are displayed in expanded form around the shock in Fig. 22. The differences at the shock are the largest, but are still quite small. No trend exists for this result; all grids produce essentially the same results. And yet if the outer grid is removed, the resulting surface pressure is dramatically in error. Thus, the outer grid serves a very important function, but its level of refinement (at least for the cases studied) is not important, as far as the surface solution is concerned. One probable explanation for this behavior is that the present solution approaches freestream very rapidly away from the cylinder surface, and although the local solution at two diameters is not that close to freestream, it is smooth enough to be reasonably captured on a coarse grid. The present solution, while being a relatively benign case (e.g., transonic airfoil flows would be more difficult), does demonstrate flexibility for the present full potential chimera approach. This latter conclusion is only valid if the surface solution is the only consideration. The off-surface flow field in the vicinity of the chimera interface for one of the coarser grids from Fig. 21 (e.g., the 17X9 or $33 \times 17$ grids) will not be accurate, especially if any kind of gradient exists at the chimera interface.

Table 3. Summary of grid statistics for the outer-zone grid refinement study, $R 2=2.46, X 1=1.0$,

\begin{tabular}{|c|c|c|c|c|c|c|c|}
\hline \multirow{3}{*}{ oasto } & \multirow{2}{*}{\multicolumn{3}{|c|}{ Grif dimensions }} & \multicolumn{4}{|c|}{ Chimera inerface arearatios } \\
\hline & & & & \multicolumn{2}{|c|}{ outer boundan. } & \multicolumn{2}{|c|}{ Sinner boundary } \\
\hline & outer & bner & blanked & $\max$ & $\min$ & $\max$ & min \\
\hline $\begin{array}{l}2017 \\
2033 \\
2065 \\
20129 \\
20257\end{array}$ & $\begin{array}{c}17 \times 9 \\
33 \times 17 \\
65 \times 33 \\
129 \times 65 \\
257 \times 129\end{array}$ & $\begin{array}{l}129 \times 49 \\
129 \times 49 \\
129 \times 49 \\
129 \times 49 \\
129 \times 49\end{array}$ & $\begin{array}{c}3 \times 2 \\
7 \times 4 \\
15 \times 8 \\
31 \times 16 \\
63 \times 32\end{array}$ & $\begin{array}{l}250 \\
51 \\
12 \\
2.9 \\
0.55\end{array}$ & $\begin{array}{l}120 \\
34 \\
9.9 \\
2.2 \\
0.72\end{array}$ & $\begin{array}{l}830 \\
110 \\
23 \\
5.2 \\
0.73\end{array}$ & $\begin{array}{r}530 \\
71 \\
14 \\
3.1 \\
1.2\end{array}$ \\
\hline
\end{tabular}

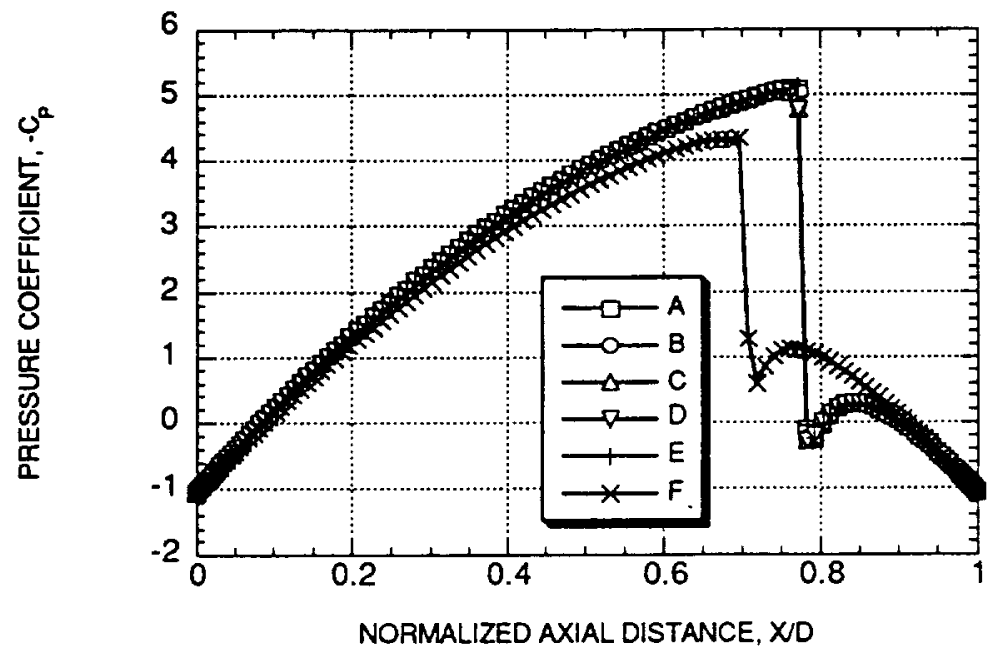

Fig. 21 Surface pressure coefficient distributions showing the effects of outer-zone grid refinement for the two-zone chimera grid arrangement, $M_{\infty}=0.5, R 2=2.46, X 1=1.0, X 2=17.5$, inner grid = $129 \times 49$ (fixed), outer grid: (A) $17 \times 9$, Case 2017, (B) 33X17, Case 2033, (C) 65X33, Case 2065, (D) 129X65, Case 20129, (E) 257X129, Case 20257, and (F) no outer grid, freestream applied directly to inner-grid outer boundary. 


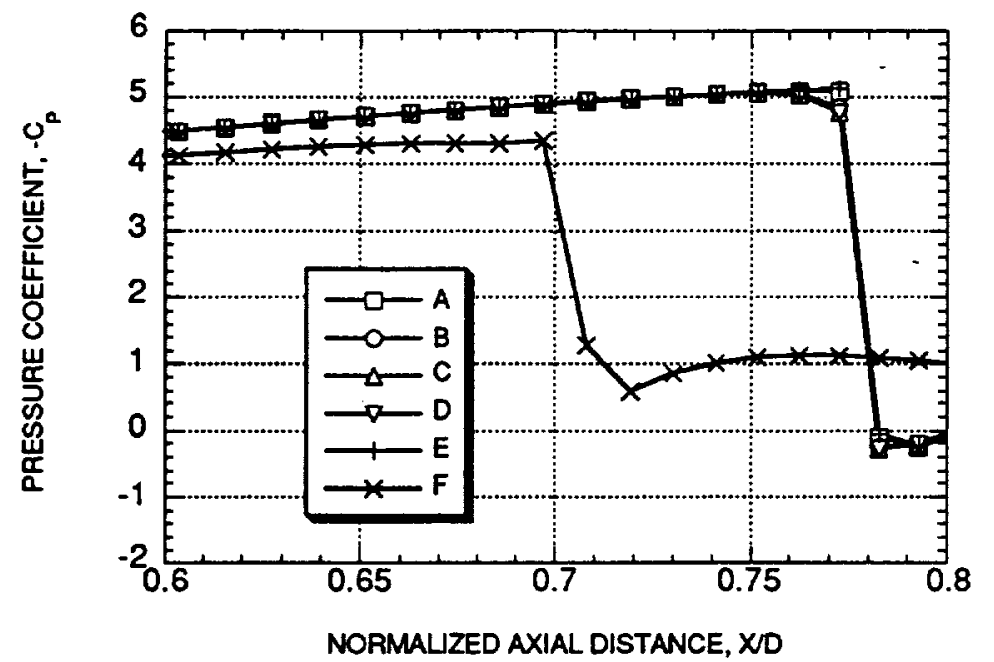

Fig. 22 Blowup view of the surface pressure coefficient distributions from Fig. 21 showing solution details around the shock wave, $M_{-}=0.5$.

\section{Chimera overlap study}

In this last result section the effect of overlap size on the solution is studied. The shocked compressible solution of the last two sections is again used. In this series of cases the outer grid dimensions are fixed at $257 \times 129$ and the inner grid dimensions are varied from $129 \times 13$ to $129 \times 49$. A summary of these grids with additional statistics is given in Table 4. For this series of cases the minimum hole boundary dimensions have again been used. The use of these smaller hole dimensions will allow the distance to the inner-grid outer boundary to be reduced to a minimum of about $\mathbf{0 . 3 3}$ diameters away from the cylinder surface. The overlap for this arrangement will reach a minimum value of about two cells.

Table 4. Summary of grid statistics for the chimera overlap refinement study, $R 2=2.46$,

\begin{tabular}{|c|c|c|c|c|c|c|}
$\mathrm{X}=0.5625, \mathrm{X}=17.5$. \\
\hline
\end{tabular}

The surface pressure coefficient distributions generated from the three grids described in Table 4 are identical to each other and to the surface pressure coefficient distributions displayed in Fig. 21, and thus, will not be displayed. Instead, results of this section are presented in the Mach number contour plot shown in Fig. 23. This computation is from the chimera grid arrangement of Table 4 with the smallest amount of overlap (Case $2 \mathrm{C} 13$ ). From this figure the grid arrangement including the overlap pattern is clearly visible. Keep in mind that the fringe points used in this computation are not plotted but are considered in computing the overlap sizes. Close inspection of the Mach number contours in this case show that this solution is virtually identical to the previous Mach number contours presented in Figs. 16 and 18. Thus, the effect of overlap on the solution for the present two-dimensional circular cylinder problem is minimal. 


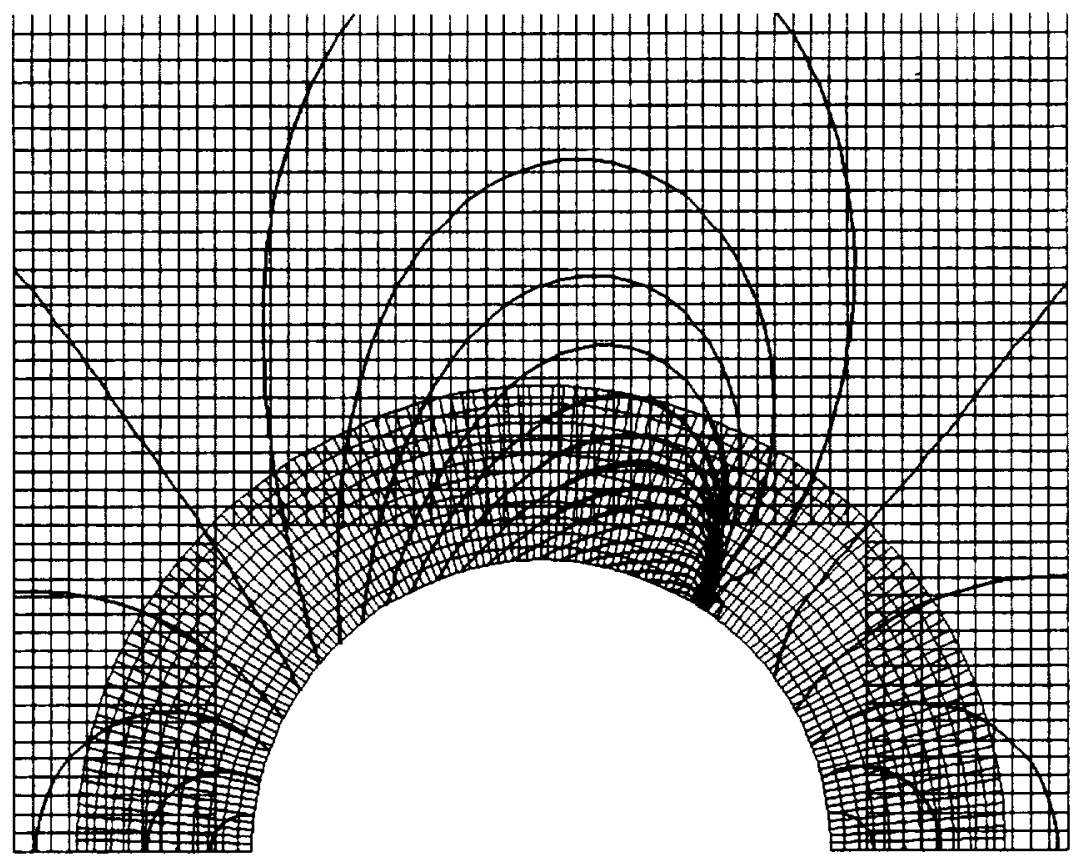

Fig. 23 Mach number contours surrounding the circular cylinder showing a minimum chimera-grid overlap condition (Case $2 \mathrm{C} 13$ from Table 4), $M_{\infty}=0.5$, inner grid $=129 \times 13$, outer gird $=257 \times 129$, $\mathrm{R} 2=0.83, \mathrm{X} 1=0.5625, \mathrm{X} 2=17.5$.

A final solution combining the effects of reduced overlap and a coarse outer grid is displayed in Fig. 24. The intermediate inner grid from Table 4 (with dimensions 129X31) and the second coarsest outer grid from Table 3 (with dimensions $33 \times 17$ ) were selected for this calculation. In order to ensure a minimum overlap of two grid cells for this case, the hole boundary was placed in the outer most location. Mach number contours about the circular cylinder are displayed in Fig. 24 showing the entire inner grid. From this figure the large disparity in grid spacing at the inner-grid outer boundary is obvious. Nevertheless, the Mach number contours from the two grids in the overlap region are nearly identical to each other. The largest discrepancy in the overlap region arises from the straight-line segments used by the plotting program to represent the contours within each outer-zone grid cell. The solution at the circular cylinder surface, including the supersonic regions and the shock wave structure, are captured with almost exactly the same level of accuracy as in Figs. 16, 18 and 23. The surface pressure coefficient distribution for this case (not shown) is also in excellent agreement with similar results (Figs. 20 and 21) computed using different grid arrangements.

\section{CONCLUDING REMARKS}

In conclusion, a chimera-based full potential algorithm has been presented and evaluated using a simple two-dimensional circular cylinder model problem. Both incompressible and shockedcompressible solutions have been used in this evaluation. The results indicate that the multi-zone chimera grid approach is a viable technique for solving the full potential equation and could provide a very fast computational tool for the aerodynamic analysis and design of geometrically complex configurations including complete aircraft. Specific conclusions are presented as follows:

A careful study of the error produced by the chimera approach was conducted using the twodimensional model problem and incompressible flow conditions. Results indicated that interpolationinduced error in the velocity potential itself was very small. Results also indicated that although the error in the pressure was larger and somewhat ill-behaved at chimera interface boundaries, it was well below the maximum error in the flow field and was not generally visible on a plot of pressure for even a moderately-coarse grid. This result was obtained when the cell area ratios at the chimera grid interface boundaries was reasonably close to unity. 
A grid refinement study produced nearly identical results from single-zone and two-zone chimera grids, both exhibiting second-order behavior over a wide range of grid refinement. Thus, the additional interpolation-induced error generated by the chimera approach does not affect the basic second-order accuracy of the present numerical scheme.

Numerous chimera-grid arrangements with widely varying overlaps and cell-interface area ratios produced remarkable accuracy (and consistency in accuracy as well) showing little variation in the solutions being studied. In particular, remarkable accuracy at the circular cylinder surface was obtained using an extremely coarse outer grid in the chimera-grid arrangement. These results were produced with the shocked-compressible solution in which a transonic shock wave passed through one of the chimera interface boundaries. The measure of error in this portion of the study focused exclusively on plotable differences in surface pressures or in Mach number contours and thus is not as precise as the error analysis in the first portion of this study, but is more representative of a practical application.

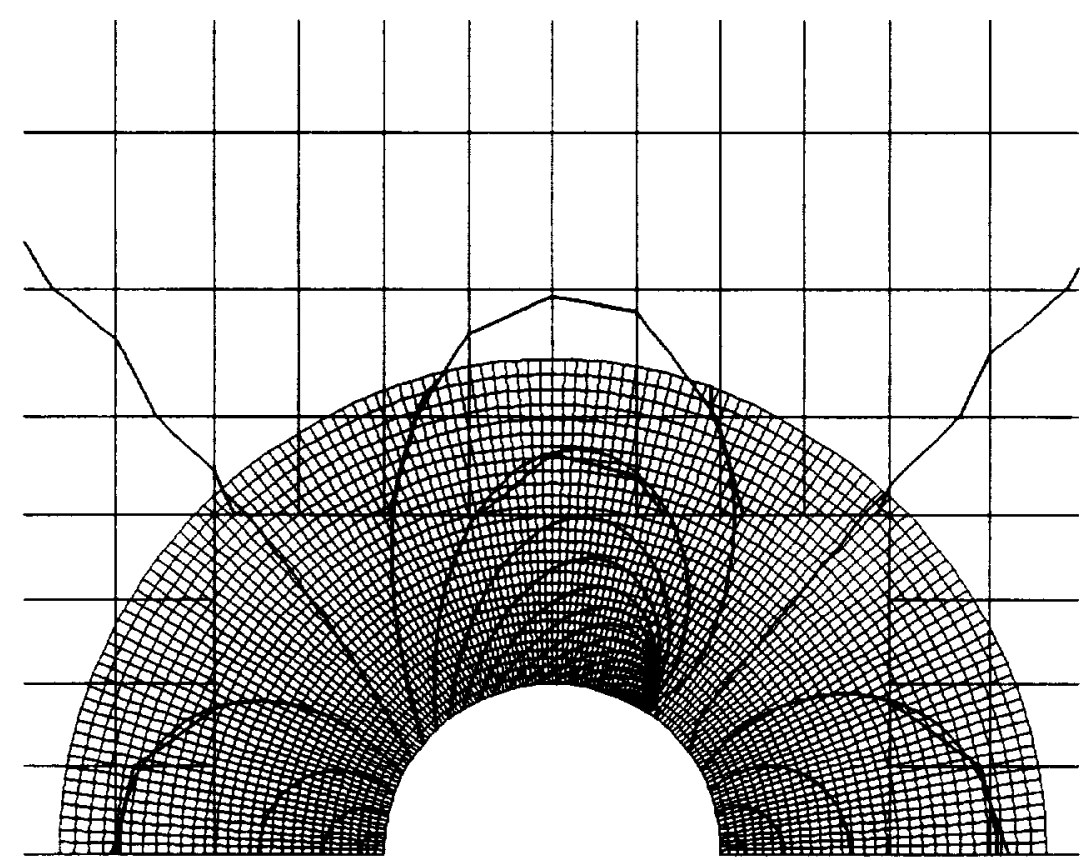

Fig. 24 Mach number contours surrounding the circular cylinder showing minimum chimera-grid overlap and coarse outer-grid conditions, $M_{\infty}=0.5$, inner grid $=129 \times 31$, outer gird $=33 \times 17, R 2=$ $1.5, \mathrm{X} 1=1.0, \mathrm{X} 2=17.5$.

\section{REFERENCES}

1. Buning, P. G., Chan, W. M., Renze, K. J., Sondak, D. L., Chiu, I.T., Slotnick, J. P., "OVERFLOW User's Manual," Unpublished NASA report, Version 1.6ad, March 1993.

2. Steger, J. L., Dougherty, F. C., and Benek, J. A., "A Chimera Grid Scheme," Advances in Grid Generation, K. Ghia and U. Ghia, Eds., ASME FED-5, 1983, pp. 59-69.

3. Benek, J. A., Steger, J. L., and Dougherty, F. C., "A Flexible Grid Embedding Technique with Application to the Euler equations," AIAA Paper No. 83-1944, July 1983.

4. Benek, J. A., Buning, P. G., and Steger, J. L., "A 3-D Grid Embedding Technique," AlAA Paper No. 85-1523, July 1985 . 
5. Dougherty, F. C., Benek, J. A., and Steger, J. L., "On Application of Chimera Grid Scheme to Store Separation to Viscous Flows," NASA TM 88193, Oct. 1985.

6. Buning, P. G., Chiu, I. T., Obayashi, S., Rizk, Y. M., and Steger, J. L., "Numerical Simulation of the Integrated Space Shuttle Vehicle in Ascent," AIAA Paper No. 88-4359-CP. Aug. 1988.

7. Buning, P. G., Chiu, I. T., Martin, F. W., Meakin, R. L., Obayashi, S., Rizk, Y. M., Steger, J. L., and Yarrow, M., "Flow field Simulation of the Space Shuttle Vehicle in Ascent," Fourth International Conference on Supercomputing, Santa Clara, CA, May 1989.

8. Martin, F. W. and Slotnick, J., "Flow Computations for the Space Shuttle in Ascent Mode Using Thin-Layer Navier-Stokes Equations," Progress in Astronautics and Aeronautics, Vol. 125, Applied Computational Aerodynamics, P. Henne, Ed., AIAA, 1990.

9. Cummings, R. M., Rizk, Y.M., Schiff, L. B., and Chaderjian, N. M., "Navier-Stokes Predictions of the Flowtield Around the F-18 (HARV) WIng and Fuselage at Large Incidence," AIAA Paper No. 90-0099, Jan. 1990.

10. Rizk, Y., Schiff, L., and Gee, K., "Numerical Simulation of the Viscous Flow Around the F-18 at High Angles of Attack," AlAA Paper No. 90-2999, Aug. 1990.

11. Murman, S. M., Rizk, Y. M., and Schiff, L. B., "Coupled Numerical Simulation of the External and Engine Inlet Flows for the F-18 at Large Incidence," AIAA Paper No. 92-2621, June 1992.

12. Murman, S. M., Schiff, L. B., and Rizk, Y. M., "Numerical Simulation of the Flow About an F-18 Aircraft in the High Alpha Regime," AIAA Paper No. 93-3405, Aug. 1993.

13. Gee, K., Rizk, Y. M., and Schiff, L. B., "Forebody Tangential Slot Blowing on an Aircraft Geometry," J. of Aircraft, Vol. 31, No. 4, July-Aug. 1994, pp. 922-928.

14. Smith, M., Chawla, K., and Van Dalsem, W., "Numerical Simulation of a Complete STOVL Aircraft in Ground Effect," AIAA Paper No. 91-3293, Sept. 1991.

15. Rizk, Y. M. and Gee, K., "Numerical Prediction of the Unsteady Flowfield Around the F-18 Aircraft at Large Incidence," AIAA Paper No. 91-0020, Jan. 1991.

16. Meakin, R., "Unsteady Aerodynamic Simulation of Multiple Bodies in Relative Motion," AIAA Paper No. 89-1996, June 1989.

17. Meakin, R., "Computations of the Unsteady Flow About a Generic Wing/Pylon/Finned-Store Configuration," AlAA Paper No. 92-4568, Aug. 1992.

18. Rizk, Y., Guruswamy, G., and Gee, K., "Numerical Investigation of Tail Buffet on F-18 Aircraft," AIAA Paper No. 92-2673, June 1992.

19. Atwood, C. A., "Unsteady Fluid and Optical Simulation of Transonic Aero-Windows," AlAA Paper No. 93-3017, July 1993.

20. Meakin, R. L., "Moving Body Overset Grid Methods for Complete Aircraft Tiltrotor Simulations," AIAA Paper No. 93-3350, July 1993.

21. Atwood, C. A. and Van Dalsem, W. R., "Flowtield Simulation About the Stratospheric Observatory for Infrared Astronomy," J. of Aircraft, Vol. 30, No. 5, Sept-Oct. 1993, pp. 719-727.

22. Lijewski, L. L. and Suhs, N., "Time-Accurate Computational Fluid Dynamics Approach to Transonic Store Separation Trajectory Prediction," J. of Aircraft, Vol. 31, No. 4, July-Aug. 1994, pp. 886-891. 
23. Atwood, C. A., "Computation of a Controlled Store Separation from a Cavity," AlAA Paper No. 940031, Jan. 1994.

24. Atta, E. H. and Vadyak, J., "A Grid Interfacing Zonal Algorithm for Three-Dimensional Transonic Flows About Aircraft Configurations, AlAA Paper 82-1017, 1982.

25. Meakin, R., "On the Spatial and Temporal Accuracy of Overset Grid Methods for Moving Body Problems," AIAA Paper No. 94-1925, June 1994.

26. Steger, J. L. and Baldwin, B. S., "Shock Waves and Drag in the Numerical Calculation of Isentropic Transonic Flow," NASA TN D-6997, 1972.

27. Flores, J., Holst, T. L., Kwak, D., and Batiste, D. M., "A New Consistent Spatial Differencing Scheme for the Transonic Full Potential Equation," AlAA J, Vol. 22, No. 8, Aug. 1984, pp. 1027.

28. Thomas, S. D. and Holst, T. L., "A Consistent Spatial Differencing Scheme for the Transonic Full Potential Equation in Three Dimensions," NASA TM-86716, Dec. 1985.

29. Holst, T. L., "Implicit Algorithm for the Conservative Transonic Full Potential Equation Using an Arbitrary Mesh," AlAAJ, Vol. 17, No. 10, Oct. 1979, pp. 1038-1045.

30. Holst, T. L. and Thomas, S. D., "Numerical Solution of Transonic Wing Flow fields," AlAA J, Vol. 21, No. 6, June 1983, pp. 863-870.

31. Ballhaus, W. F., Jameson, A., and Albert, J., "Implicit Approximate Factorization Schemes for the Efficient Solution of Steady Transonic Flow Problems," AlAA J, Vol. 16, June 1978, pp. 573-579.

32. South, J. C., Jr. and Hafez, M. M., "Stability Analysis of Intermediate Boundary Conditions in Approximate Factorization Schemes," AIAA Paper No. 83-1898, July 1983.

33. Walatka, P. P., Buning, P. G., Pierce, L., and Elson, P. A., "PLOT3D User's Manual," NASA TM 101067, July 1992. 


\section{AGENCY USE ONLY (Leave blank) \\ 2. REPORT DATE \\ 3. REPORT TYPE AND DATES COVERED \\ July 1995 \\ Technical Memorandum}

4. TITLE AND SUBTITLE

Numerical Solution of the Full Potential Equation Using a

Chimera Grid Approach

6. AUTHOR(S)

$505-59-53$

Terry L. Holst

7. PERFORMING ORGANIZATION NAME(S) AND ADDRESS(ES)

8. PERForming ORGANIZATION REPORT NUMBER

Ames Research Center

Moffett Field, CA 94035-1000

A-950082

9. SPONSORING/MONITORING AGENCY NAME(S) AND ADDRESS(ES)

10. SPONSORING/MONITORING AGENCY REPORT NUMBER

National Aeronautics and Space Administration

Washington, DC 20546-0001

NASA TM-110360

11. SUPPLEMENTARY NOTES

Point of Contact: Terry L. Holst, Ames Research Center, MS T27B-1, Moffett Field, CA 94035-1000 (415) 604-6032

12a. DISTRIBUTION/AVAILABILITY STATEMENT

12b. DISTRIBUTION CODE

Unclassified-Unlimited

Subject Category - 02

13. ABSTRACT (Maximum 200 words)

A numerical scheme utilizing a chimera zonal grid approach for solving the full potential equation in two spatial dimensions is described. Within each grid zone a fully-implicit approximate factorization scheme is used to advance the solution one iteration. This is followed by the explicit advance of all common zonal grid boundaries using a bi-linear interpolation of the velocity potential. The presentation is highlighted with numerical results simulating the flow about a two-dimensional, nonlifting circular cylinder. For this problem, the flow domain is divided into two parts: an inner portion covered by a polar grid and an outer portion covered by a Cartesian grid. Both incompressible and compressible (transonic) flow solutions are included. Comparisons made with an analytic solution as well as single grid results indicate that the chimera zonal grid approach is a viable technique for solving the full potential equation.

\section{SUBJECT TERMS}

15. NUMBER OF PAGES

Numerical methods, Transonic flow, Chimera, Inviscid flow 
